\title{
Hypergraph partitioning for multiple communication cost metrics: Model and methods
}

\author{
Mehmet Deveci ${ }^{\mathrm{a}, \mathrm{c}, *}$, Kamer Kaya ${ }^{\mathrm{c}, \mathrm{b}},{\text { Bora } \mathrm{Uçar}^{\mathrm{d}} \text {, Ümit V. Çatalyürek }}^{\mathrm{c}, \mathrm{e}}$ \\ ${ }^{a}$ Dept. of Computer Science and Engineering, The Ohio State University, Columbus OH, \\ USA \\ ${ }^{b}$ Computer Science and Engineering, Sabancı University, Istanbul, Turkey \\ ${ }^{c}$ Dept. of Biomedical Informatics, The Ohio State University, Columbus OH, USA \\ ${ }^{d}$ CNRS and LIP, ENS Lyon, France \\ ${ }^{e}$ Dept. of Electrical and Computer Engineering, The Ohio State University, Columbus OH, \\ $U S A$
}

\begin{abstract}
We investigate hypergraph partitioning-based methods for efficient parallelization of communicating tasks. A good partitioning method should divide the load among the processors as evenly as possible and minimize the inter-processor communication overhead. The total communication volume is the most popular communication overhead metric which is reduced by the existing state-of-theart hypergraph partitioners. However, other metrics such as the total number of messages, the maximum amount of data transferred by a processor, or a combination of them are equally, if not more, important. Existing hypergraphbased solutions use a two phase approach to minimize such metrics where in each phase, they minimize a different metric, sometimes at the expense of others. We propose a one-phase approach where all the communication cost metrics can be effectively minimized in a multi-objective setting and reductions can be achieved for all metrics together. For an accurate modeling of the maximum volume and the number of messages sent and received by a processor, we propose the use of directed hypergraphs. The directions on hyperedges necessitate revisiting the standard partitioning heuristics. We do so and propose a multi-objective, multi-level hypergraph partitioner called UMPa. The partitioner takes various prioritized communication metrics into account, and optimizes all of them together in the same phase. Compared to the state-of-the-art methods which only minimize the total communication volume, we show on a large number of problem instances that UMPa produces better partitions in terms of several communication metrics.
\end{abstract}

\footnotetext{
* Corresponding author

Email addresses: mdeveci@bmi.osu.edu (Mehmet Deveci), kaya@sabanciuniv.edu (Kamer Kaya), bora.ucar@ens-lyon.fr (Bora Uçar), umit@bmi.osu.edu (Ümit V. Çatalyürek)
} 


\section{Introduction}

Finding a good partition of communicating tasks among the available processing units is crucial for obtaining short execution times, using less energy, and utilizing the computation and communication resources better. To solve this problem, several graph and hypergraph models have been proposed $[1,2,3$, $4,5,6]$. These models transform the problem at hand to a balanced partitioning problem. The balance restriction on part weights in conventional partitioning corresponds to the load balance in a parallel environment, and the minimization objective for a given metric relates to the minimization of the communication between the processing units.

The most widely used communication metric is the total communication volume. Other communication metrics, such as the total number of messages [7], the maximum volume of messages sent and/or received by a processor [7, 8], or the maximum number of messages sent by a processor have also been shown to be important. The latency-based metrics, which model the communication by using the number of messages sent/received throughout the execution, become more and more important as the number of processors increases. Ideal partitions yield perfect computational load balance and minimize the communication requirements by minimizing all the mentioned metrics.

Given an application, our main objective is to partition the tasks evenly among processing units and to minimize the communication overhead by minimizing several communication cost metrics. Previous studies addressing different communication cost metrics (such as $[7,8]$ ) work in two phases where the phases are concerned with disjoint subsets of communication cost metrics. We present a novel approach to treat the minimization of multiple communication metrics as a multi-objective minimization in a single phase. In order to achieve that, we propose the use of directed hypergraph models. We have materialized our approach in UMPa (pronounced as "Oompa"), which is a multi-level partitioner employing a directed hypergraph model and novel $K$-way refinement heuristics. In an earlier work [9], we had presented how minimization of the maximum communication volume can be modeled. Here, we extend that work for a more generic framework. UMPa not only takes the total and the maximum communication metric into account, but it also treats the total and maximum number of messages in a more generalized framework. It aims to minimize the primary metric and obtains improvements in the secondary communication metrics. Compared to the state of the art partitioning tools PaToH [10], Mondriaan [11], and Zoltan [12] using the standard hypergraph model, which minimize the total communication volume, we show on a large number of problem instances that UMPa produces much better partitions in terms of several communication metrics with $128,256,512$, and 1024 processing units.

The organization of the paper is as follows. In Section 2, the background material on the hypergraph partitioning is given. We also summarize the previous work on minimizing multiple communication cost metrics in this section. Section 3 presents the directed hypergraph model, and explains how the communication metrics are encoded by those hypergraphs. In Section 4, we present our 
multi-level, multi-objective partitioning tool UMPa and give its implementation details in Section 5. Section 6 presents the experimental results, and Section 7 concludes the paper.

\section{Background}

\subsection{Hypergraph partitioning}

A hypergraph $\mathcal{H}=(\mathcal{V}, \mathcal{N})$ is defined as a set of vertices $\mathcal{V}$ and a set of nets (hyperedges) $\mathcal{N}$ among those vertices. A net $n \in \mathcal{N}$ is a subset of vertices and the vertices in $n$ are called its pins. The number of pins of a net is called the size of it, and the degree of a vertex is equal to the number of nets it belongs to. We use pins $[n]$ and nets $[v]$ to represent the pin set of a net $n$, and the set of nets containing a vertex $v$, respectively. Vertices can be associated with weights, denoted with w[·], and nets can be associated with costs, denoted with c[·].

A $K$-way partition of a hypergraph $\mathcal{H}$ is a partition of its vertex set, which is denoted as $\Pi=\left\{\mathcal{V}_{1}, \mathcal{V}_{2}, \ldots, \mathcal{V}_{K}\right\}$, where

- parts are pairwise disjoint, i.e., $\mathcal{V}_{k} \cap \mathcal{V}_{\ell}=\emptyset$ for all $1 \leq k<\ell \leq K$,

- each part $\mathcal{V}_{k}$ is a nonempty subset of $\mathcal{V}$, i.e., $\mathcal{V}_{k} \subseteq \mathcal{V}$ and $\mathcal{V}_{k} \neq \emptyset$ for $1 \leq k \leq K$,

- the union of $K$ parts is equal to $\mathcal{V}$, i.e., $\bigcup_{k=1}^{K} \mathcal{V}_{k}=\mathcal{V}$.

Let $W_{k}$ denote the total vertex weight in $\mathcal{V}_{k}$, that is $W_{k}=\sum_{v \in \mathcal{V}_{k}} \mathrm{w}[v]$, and $W_{\text {avg }}$ denote the weight of each part when the total vertex weight is equally distributed, that is $W_{a v g}=\sum_{v \in \mathcal{V}} \mathrm{w}[v] / K$. If each part $\mathcal{V}_{k} \in \Pi$ satisfies the balance criterion

$$
W_{k} \leq W_{a v g}(1+\varepsilon), \quad \text { for } k=1,2, \ldots, K
$$

we say that $\Pi$ is $\varepsilon$-balanced where $\varepsilon$ is called the maximum allowed imbalance ratio.

For a $K$-way partition $\Pi$, a net that has at least one pin (vertex) in a part is said to connect that part. The number of parts connected by a net $n$ is called its connectivity and denoted as $\lambda_{n}$. A net $n$ is said to be uncut (internal) if it connects exactly one part (i.e., $\lambda_{n}=1$ ), and cut (external), otherwise (i.e., $\left.\lambda_{n}>1\right)$. Given a partition $\Pi$, if a vertex is in the pin set of at least one cut net, it is called a boundary vertex.

In the text, we use part $[v]$ to denote the part of vertex $v$ and $\operatorname{prts}[n]$ to denote the set of parts net $n$ is connected to. Let $\Lambda(n, p)=\left|\operatorname{pins}[n] \cap \mathcal{V}_{p}\right|$ be the number of pins of net $n$ in part $p$. Hence, $\Lambda(n, p)>0$ if and only if $p \in \operatorname{prts}[n]$.

There are various cutsize definitions [13] for hypergraph partitioning. The one which is widely used in the literature and shown to accurately model the total communication volume of parallel sparse matrix-vector multiplication [2] is called the connectivity- 1 metric. This cutsize metric is defined as: 


$$
\chi(\Pi)=\sum_{n \in \mathcal{N}} \mathrm{c}[n]\left(\lambda_{n}-1\right)
$$

In this metric, each cut net $n$ contributes c $[n]\left(\lambda_{n}-1\right)$ to the cutsize. The hypergraph partitioning problem can be defined as the task of finding a balanced partition $\Pi$ with $K$ parts such that $\chi(\Pi)$ is minimized. This problem is NPhard [13].

\subsection{Multi-level framework and partitioning}

The multi-level approach has been shown to be the most successful heuristic for various graph/hypergraph partitioning problems $[2,14,15,16,17,18]$. In the multi-level approach, a given hypergraph is successively (i.e., level by level) coarsened to a much smaller one, a partition is obtained on the smallest hypergraph, and that partition is successively projected to the original hypergraph while being improved at each level. These three phases are called the coarsening, initial partitioning, and uncoarsening phases, respectively. In a coarsening level, similar vertices are merged into a single vertex, reducing the size of the hypergraph at each level. In the corresponding uncoarsening level, the merged vertices are split, and the partition of the coarser hypergraph is refined for the finer one using Kernighan-Lin (KL) [19] and Fiduccia-Mattheyses (FM) [20] based heuristics.

Most of the multi-level partitioning tools used in practice, such as the sequential partitioning tools like $\mathrm{PaToH}[10]$ and hypergraph partitioning implementation of Mondriaan [11], and parallel hypergraph partitioning implementation of Zoltan [12], are based on recursive bisection. In recursive bisection, the multilevel approach is used to partition a given hypergraph into two. Each of these parts is further partitioned into two recursively until $K$ parts are obtained in total. Hence, to partition a hypergraph into $K=2^{k}$, the recursive bisection approach uses $K-1$ coarsening, initial partitioning, and uncoarsening phases.

A direct $K$-way partitioning approach within the multi-level framework is also possible [18, 17] Given the hypergraph, the partitioner gradually coarsens it in a single coarsening phase and then partitions the coarsest hypergraph directly into $K$ parts in the initial partitioning phase. Starting with this initial partition, at each level of the uncoarsening phase, the partitioner applies a $K$ way refinement heuristic after projecting the partition of the coarser hypergraph to the finer one.

\subsection{Standard hypergraph partitioning models}

The total amount of data transfer throughout the execution of the tasks is called the total communication volume (TV). Given an application with interacting tasks, in the traditional hypergraph model, the tasks, interactions, and processors correspond to vertices, nets, and parts, respectively. In this model, when a net $n$ is connected to multiple parts, a communication of size $c[n]$ from one of those parts to others (i.e., to $\lambda_{n}-1$ parts) is required, hence resulting in a 
communication cost of $\mathrm{c}[n]\left(\lambda_{n}-1\right)$. Hence, the connectivity-1 metric $(2.2)$ corresponds exactly to the total communication volume [2]. Note that this metric is independent from the direction of the interactions.

The traditional undirected hypergraph model is used for circuit partitioning in VLSI layout design [13]. It is also widely used to model various scientific computations such as the sparse-matrix vector multiplication ( $\mathrm{SpMxV})[2,3,11]$. There are three basic models for partitioning a matrix by using a hypergraph: column-net, row-net, and fine-grain. In the column-net model, the rows and the columns are represented with vertices and nets, respectively [2]. It is vice versa for the row-net model. In the fine grain model, the nonzeros of the matrix correspond to the vertices and the rows and columns correspond to the nets [21]. A one-dimensional row (column) partitioning is applied when the matrix is modeled with a column-net (row-net) hypergraph. For the fine-grain model, a $2 \mathrm{D}$ partitioning of the nonzeros is employed. We refer the reader to [3] for further information and comparative evaluation of these models.

\subsection{Related work and contributions}

Although minimizing the total communication volume TV is important, it is sometimes preferable to reduce other communication metrics [4]. The previous studies on minimizing multiple communication cost metrics are based on twophase approaches. Generally, the first phase tries to obtain a proper partition of data for which the total communication volume is reduced. Starting from the partition of the first phase, the second phase tries to optimize another communication metric. Such two-phase approaches could allow the use of stateof-the-art techniques in one or both of the phases. However, they could get stuck in some local optima that it cannot be improved in the other phase, as the solutions that are sought in one phase are oblivious to the metric used in the other phase.

Bisseling and Meesen [8] discuss how to reduce the maximum send and receive volume per processor in the second phase, while keeping the total volume of communication intact. This is achieved by a greedy assignment algorithm that assigns a data source to a processor that needs it and that has the smallest send/receive volume under current assignments. Bisseling and Meesen also discuss a greedy improvement algorithm applied after the assignments are done.

Uçar and Aykanat [7] discuss how to reduce the total number of messages and achieve balance on the maximum volume of messages sent by a processor as a hypergraph partitioning problem in the second phase. The balance on the volume of messages sent by a processor is achieved only approximately (the proposed model does not encode the send volume of a processor exactly). The metric of the maximum number of messages sent by a processor is somehow incorporated into the second phase as well. Both these second phase alternatives, however, can increase the total volume of communication found in the first phase. The amount of increase is bounded by the number of cut nets found in the first phase.

Both of the mentioned studies [7, 8] consider applications where some input data is combined to yield the output data, as in the sparse matrix-vector multi- 
ply operation $\mathbf{y} \leftarrow \mathbf{A x}$. In such settings, sometimes it is advisable to align the partition on the input and output vectors, e.g., in $\mathbf{y} \leftarrow \mathbf{A x}$ the processor that holds an $\mathbf{x}_{i}$ holds the corresponding entry $\mathbf{y}_{i}$. This latter requirement is called symmetric vector partitioning and cannot be satisfied easily with the methods proposed in these two studies.

Uçar and Aykanat [22] discuss how to extend their earlier approach to address the symmetric partitioning requirement as well for the computations of the form $\mathbf{y} \leftarrow \mathbf{A x}$. Their approach can only handle the cases where one has the liberty to partition the vector entries independent of the matrix partition. In their work, Uçar and Aykanat show how this liberty arises when A is partitioned on the nonzero basis. Again, the second phase can increase the total volume of communication found in the first phase where a non-trivial upper bound on the amount of increase is known. In some other cases, for example, when the matrix is to be partitioned rowwise and the owner computes rule has to be respected, then the method is not applicable. The entry $\mathbf{y}_{i}$ should be computed at the processor holding the $i$ th row of $\mathbf{A}$ (this, in turn, determines the partition on $\mathbf{x})$, unless of course one is ready to pay for another communication round.

Our main contribution in this work is to address multiple communication cost metrics in a single phase. Addressing all the metrics in a single phase would allow trading off the cost associated with one metric in favor of that associated with another one. The standard hypergraph model cannot see the communication metrics that are defined on a per-processor basis, therefore balance on communication loads of the processors cannot be formulated naturally. Furthermore, since all the state-of-the-art partitioners use iterative-improvement-based heuristics for the refinement, a single-phase approach increases search space by avoiding to get stuck in a local optimum for a single metric. In order to overcome these obstacles, we propose to use directed hyperedges and minimize a prioritized set of metrics all together. We associate each input and output data and computational tasks with a vertex as is done in the standard hypergraph models [23]. We then encode the dependencies among the data and computational tasks with directed hyperedges so that a unique source or a destination is defined for each hyperedge. This way, we are able to accurately model the total as well as per-processor communication cost metrics and reduce all metrics together. Furthermore, this allows the optimization of the communication cost metrics both in unsymmetric and symmetric input and output data partitions constraints, by incorporating the vertex amalgamation technique discussed in [23]. Directions on hyperedges necessitate revisiting some parts of the standard multi-level partitioning heuristics. We do so and realize our communication cost minimizing methods in a multi-objective, multi-level hypergraph partitioner called UMPa. As balancing the per-processor communication cost metrics requires a global view of the partition, we design a direct $K$-way refinement heuristic. One more positive side effect of using the proposed directed model, as we will demonstrate in the experimental evaluation section, is that one could further reduce a primary metric when additional secondary and tertiary metrics (related to the communication) are given to UMPa. 


\section{The directed hypergraph model}

Let $\mathcal{A}=(\mathcal{T}, \mathcal{D})$ be an application where $\mathcal{T}$ is the set of tasks to be executed and $\mathcal{D}$ the set of data elements processed during the application. The tasks may have different execution times; for a task $t \in \mathcal{T}$, we use exec(t) to denote its execution time. The data elements may have different sizes; for a data element $d \in \mathcal{D}$, we use size $(d)$ to denote its size. Data elements can be input and output elements, hence, respectively, they may not have any producer or consumer task in the application, or they can be produced by some tasks and consumed/used by other tasks. Graphs, and their variants, such as the standard and bipartite graphs [4], directed acyclic graphs [24], and hypergraphs [3] have been used to model many such applications with different dependency constraints.

Here, we are interested in a set of applications that can be executed in parallel similar to the bulk synchronous parallel (BSP) model of execution $[25,26]$. In other words, the set of tasks will be executed concurrently on a set of processors, consume and produce data elements, and then they will exchange data among each other. This process is usually repeated, and the same computation and communication patterns are realized in multiple iterations. To ensure that the data dependencies are met, one can either have an explicit barrier synchronization between each iteration, as in the original BSP model, or a more complex scheduling can be used in each processor that would delay the execution of the tasks for which input data elements have not been received yet. Such an execution model fits well to many scientific computations [26], iterative solvers, and also to generalization of other execution models, such as parallel reductions [27], MapReduce [28], and its iterative [29] and pipelined [30] variants.

We assume the owner-computes rule: each task $t \in \mathcal{T}$ is executed by the processing unit to which $t$ is assigned. Consider an iterative solver for a linear system of equations (such as the conjugate gradients) that repeatedly performs $\mathbf{y} \leftarrow \mathbf{A x}$ and applies linear vector operations on $\mathbf{x}$ and $\mathbf{y}$. One way to parallelize such a solver is to use rowwise matrix partitioning and assign sets of matrix rows and corresponding $\mathbf{y}$ and $\mathbf{x}$ elements to the processing units $[2,26]$. In such an assignment, the atomic task $t_{i}$ is defined as the computation of the inner product of the $i$-th row with the $\mathbf{x}$ vector, i.e., $\mathbf{y}_{i} \leftarrow \mathbf{A}_{i *} \mathbf{x}$, where data element $\mathbf{y}_{i}$ is produced by task $t_{i}$.

We assume that during the application execution, the producers of the data elements send data to their consumers. If they exist, the consumers of a data element from $\mathcal{D}$ are all in $\mathcal{T}$. In some applications, there is a direct one-to-one mapping of the data elements and tasks. That is, task $t_{i}$ produces data element $d_{i}$. However, in general, there can be data in $\mathcal{D}$ which are given as input and may not be produced by a task.

\subsection{Modeling the application}

We propose modeling the application with a directed hypergraph [31]. Given an application $\mathcal{A}$, we construct the directed hypergraph $\mathcal{H}=(\mathcal{V}, \mathcal{N})$ as follows. For each task $t_{i} \in \mathcal{T}$, we have a corresponding vertex $v_{i} \in \mathcal{V}$. For each data 
element $d_{j} \in \mathcal{D}$, we have a corresponding vertex $v_{j} \in \mathcal{V}$ and a corresponding net $n_{j} \in \mathcal{N}$, where

$$
\mathrm{w}[v]= \begin{cases}\operatorname{exec}(t) & \text { if } v \text { corresponds to a task } t \\ 0 & \text { if } v \text { corresponds to a data item. }\end{cases}
$$

and $\mathrm{c}\left[n_{j}\right]=\operatorname{size}\left(d_{j}\right)$. Since we are interested in balancing the computational load of the processors, the vertices corresponding to data items have zero weight. If one also wants to balance the storage, which may be necessary for memoryrestricted processing units, a multiconstraint hypergraph partitioning scheme [32] can be used with extra positive weights, representing size of data elements, on these vertices and zero weights on the vertices corresponding to tasks.

The pins of a net $n_{j}$, pins $\left[n_{j}\right]$, include a producer (also called source), which will be denoted as $\operatorname{src}\left[n_{j}\right]$, and consumers of the corresponding data item $d_{j}$. In this directed hypergraph model, the communication represented by a net $n$ is flowing from its producer vertex to its consumer vertices pins $[n] \backslash\{\operatorname{src}[n]\}$.

Figure 1a shows the sparse matrix and vectors of the $\mathrm{SpMxV}$ operation $\mathbf{y} \leftarrow$ Ax in a sample of the iterative solver application mentioned above. Figure 1b shows the associated directed hypergraph model in the case where a rowwise partitioning is required. In the model, there are three types of vertices $\left(x_{i}, y_{i}\right.$, and $r_{i}$ for $i=1, \ldots, 6$ ), each modeling an $\mathbf{x}$ - or a $\mathbf{y}$-vector entry, or a row of A. The vertices are shown as labeled white circles. The nets are shown as filled, small circles. The directions of the pins are set from $x$-vertices to the corresponding nets, from those nets to the row vertices. The $y$-vertices are the destinations of the corresponding nets whose sources are the corresponding row vertices.

In the directed hypergraph model of Figure 1b, there is a single sender and multiple receivers for each net. This model fits well with many scientific applications where there is a single owner of the data, who is also responsible for distributing the updated value to processors that needs it. There are applications in which there are multiple senders that send, usually, their partial updates to the owner of the data. Some other applications use both of these models iteratively, such as gathering of partial updates followed by distribution of the updated values (e.g., [33]). A directed hypergraph can model such applications. However, the current implementation of UMPa supports only the discussed applications with a single sender. We note that in $\mathrm{SpMxV}$ when the partitioning is columnwise or on nonzero basis (that is a two dimensional partitioning on the matrix), some nets of the directed hypergraph will have multiple senders, in which case UMPa cannot be used in its current form.

In our directed hypergraph model, a part $p$ corresponds to a processing unit $p$, and the balance restriction of the partitioning problem on the part weights necessitates a balanced distribution of the computational load among the processing units. In addition, the total communication volume corresponds to the connectivity-1 metric in (2.2). 


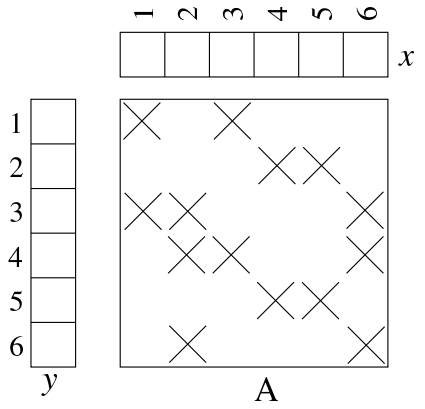

(a) Sample matrix-vector multiplication

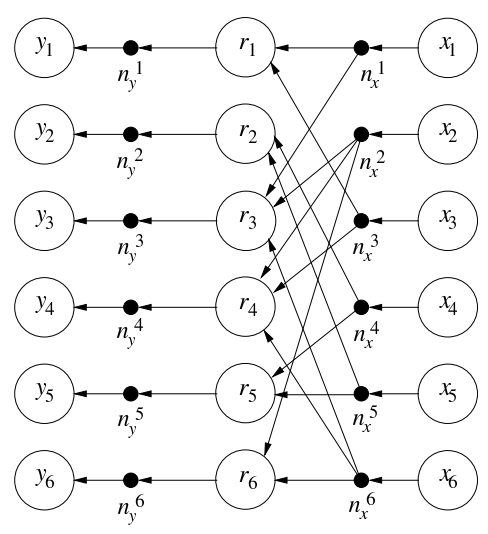

(b) Directed hypergraph model

Figure 1: Sample sparse matrix-vector multiplication and the corresponding directed hypergraph model.

\subsection{Communication cost metrics using direction information}

Given a $K$-way partition, let $\mathrm{SV}[p]$ and $\mathrm{RV}[p]$ be the data volume the processing unit $p$ sends and receives, respectively. That is

$$
\begin{aligned}
& \mathrm{SV}[p]=\sum_{\operatorname{part}[\operatorname{src}[n]]=p} \mathrm{c}[n]\left(\lambda_{n}-1\right), \\
& \mathrm{RV}[p]=\sum_{\substack{\operatorname{part}[\operatorname{src}[n]] \neq p \\
p \in \operatorname{prts}[n]}} \mathrm{c}[n] .
\end{aligned}
$$

Hence, TV equals $\sum_{i=1}^{K} \mathrm{SV}\left[p_{i}\right]=\sum_{i=1}^{K} \mathrm{RV}\left[p_{i}\right]$. Let $\mathrm{SRV}[p]=\mathrm{SV}[p]+\mathrm{RV}[p]$ be the total volume of data sent/received by the processor $p$. The maximum data volume sent MSV and sent/received MSRV by a single processor are defined as

$$
\begin{aligned}
\mathrm{MSV} & =\max _{i}\left\{\mathrm{SV}\left[p_{i}\right]\right\}, \\
\mathrm{MSRV} & =\max _{i}\left\{\operatorname{SRV}\left[p_{i}\right]\right\} .
\end{aligned}
$$

Let $\operatorname{SM}\left[p_{i}\right]$ be the number of messages the processing unit $p_{i}$ sends, that is

$$
\mathrm{SM}\left[p_{i}\right]=\mid\left\{p_{j}: \exists n \text { s.t. } \operatorname{part}[\operatorname{src}[n]]=p_{i} \text { and } p_{j} \in \operatorname{prts}[n] \backslash\left\{p_{i}\right\}\right\} \mid .
$$

The total number of messages TM, and the maximum number of messages sent by a single processor MSM are defined as

$$
\begin{aligned}
\mathrm{TM} & =\sum_{i} \mathrm{SM}\left[p_{i}\right], \\
\mathrm{MSM} & =\max _{i}\left\{\mathrm{SM}\left[p_{i}\right]\right\} .
\end{aligned}
$$




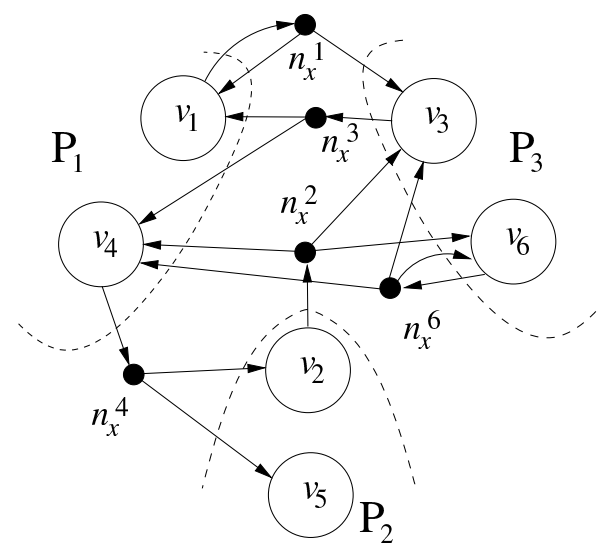

Figure 2: A 3-way partition of the simplified directed hypergraph. A vertex $v_{i}$ now represents $x_{i}, y_{i}$ and row $r_{i}$. Among the nets, only the ones that are cut are shown.

Consider again the sparse matrix-vector multiplication of Fig. 1 in the context of an iterative solver where the vectors $\mathbf{x}$ and $\mathbf{y}$ undergo linear operations (such as $\mathbf{x}_{i} \leftarrow \mathbf{x}_{i}+\beta \mathbf{y}_{i}$ for a scalar $\beta$ to form the $\mathbf{x}$ of the next iteration). In this case, the hypergraph can be simplified by using a set of modifications which are useful to avoid some extra communications. First, the data items $\mathbf{x}_{i}$ and $\mathbf{y}_{i}$ should be in the same part upon partitioning - otherwise an extra data transfer is required during the vector operations. Second, $\mathbf{y}_{i}$ is produced by $r_{i}$; unless they are in the same part, an extra communication is required. It is therefore advisable to combine the vertices $x_{i}, y_{i}$, and $r_{i}$ for all $i$ (see the vertex amalgamation operation introduced in [7]; see also [23]). This simplified model corresponds to the column-net hypergraph model [2]. Consider the 3 -way partition of the resulting hypergraph depicted in Fig. 2 (internal nets are not shown for clarity). Under this partition, the processor $p_{1}$, corresponding to the part $P_{1}$, holds vector entries $x_{1}, x_{4}$, rows $r_{1}, r_{4}$ and is responsible for computing $y_{1}$ and $y_{4}$ at the end of multiplication. Similar data and computation assignments apply to the processors $p_{2}$ and $p_{3}$ corresponding to the parts $P_{2}$ and $P_{3}$. As seen with the directions on the pins of the cut nets, processor $p_{1}$ sends $x_{1}$ to $p_{3}$ and $x_{4}$ to $p_{2}$; processor $p_{2}$ sends $x_{2}$ to $p_{1}$ and $p_{3}$; and $p_{3}$ sends $x_{3}$ and $x_{6}$ to $p_{1}$. Combining these, we see that $\mathrm{SV}\left[p_{i}\right]=2$, for all $i ; \operatorname{RV}\left[p_{1}\right]=3, \operatorname{RV}\left[p_{2}\right]=1$, and $\mathrm{RV}\left[p_{3}\right]=2$. We further note that $\mathrm{SM}\left[p_{1}\right]=\operatorname{SM}\left[p_{2}\right]=2, \operatorname{SM}\left[p_{3}\right]=1$.

The directions on the hyperedges help to quantify the sends and receives of each processor. Without directions, one would not know, for example, if the information flow on $n_{x}^{3}$ should have been as described. If there were no vertex amalgamation, it would be possible to compute these without directions, but with a little bookkeeping and additional computation. However, vertex amalgamation type operations (e.g., the coarsening phase in a multi-level partitioner) 
always take place in the state-of-the-art partitioners. In this case, quantifying the sends and receives of each processor would require too much bookkeeping (the contents of a composite vertex and connections of the composing vertices to the nets) and too much computation to be useful in the multi-level partitioning framework. The directions in the nets avoid this difficulty, but necessitate the development of suitable partitioning tools.

\section{UMPa: A multi-objective partitioning tool for communication min- imization}

The proposed partitioner, UMPa, aims to optimize a given volume- or latencybased primary metric and tries to reduce a set of secondary communication metrics. Although the recursive bisection approach can work well for the total communication volume metric (TV), it is not suitable for the maximum volume (MSV), the total number of messages (TM), and the maximum number of messages sent by a processor (MSM). Since we aim to handle multiple communication metrics all together, UMPa follows the direct $K$-way partitioning approach.

\subsection{Multi-level coarsening phase}

In this phase, the original hypergraph is gradually coarsened in multiple levels by clustering subsets of vertices at each level. There are two types of clustering algorithms: matching-based and agglomerative. The matching-based algorithms put at most two similar vertices in a cluster, whereas the agglomerative ones allow any number of similar vertices. There are various similarity metrics - see for example $[10,34,12]$. All these metrics are defined only on two adjacent vertices (one of them can be a vertex cluster). Two vertices are adjacent if they share a net and they can be in the same cluster if they are adjacent.

We use an agglomerative algorithm and the absorption clustering metric using pins $[10,34]$. For this metric, the similarity between two adjacent vertices $u$ and $v$ is

$$
\sum_{n \in \operatorname{nets}[u] \cap \operatorname{nets}[v]} \begin{gathered}
\mathrm{c}[n] \\
|\operatorname{pins}[n]|-1
\end{gathered}
$$

This is also the default metric in $\mathrm{PaToH}[10]$. In each level $\ell$, we start with a finer hypergraph $\mathcal{H}^{\ell}$ and obtain a coarser one $\mathcal{H}^{\ell+1}$. If $\mathcal{V}_{C} \subset \mathcal{V}^{\ell}$ is a subset of vertices deemed to be clustered, we create the cluster vertex $u \in \mathcal{V}^{\ell+1}$ where nets $[u]=\cup_{v \in \mathcal{V}_{C}}$ nets $[v]$. We also update the pin sets of the nets in nets $[u]$ accordingly.

Since we need the direction, we always store the source vertex of a net $n \in \mathcal{N}$ as the first pin in pins $[n]$. To maintain this information, when a cluster vertex $u$ is formed in the coarsening phase, we put $u$ to the head of pins $[n]$ for each net $n$ whose source vertex is in the cluster. We also discard the nets that become singleton at each step of the coarsening phase as well as the initial singleton nets, since they do not cause any communication. 


\subsection{Initial partitioning phase}

To obtain an initial partition for the coarsest hypergraph, we use $\mathrm{PaToH}[10]$, which is proved to produce high quality partitions with respect to the total communication volume [2]. We execute $\mathrm{PaToH}$ five times and get the best partition according to the given primary metric. We chose to use $\mathrm{PaToH}$ for three reasons. First, since we always take TV into account either as a primary or a secondary metric, it is better to start with an initial partition having a good total communication volume. Second, since TV is the sum of the send volumes of all parts, minimizing it should also be good for both MSV and MSRV and even for the latency-based metrics. We verified the second reason, although it sounds intuitive, in preliminary experiments [9]. The third reason is more esoteric. The coarsest hypergraph which has small net sizes and high vertex degrees lends itself gracefully to the recursive bisection and FM-based improvement heuristics (see also elsewhere [18]).

\subsection{Uncoarsening phase and one-phase $K$-way refinement}

The uncoarsening phase is realized in multiple levels corresponding to the coarsening levels where at the $\ell$ th level, we project the partition $\Pi^{\ell+1}$ obtained for $\mathcal{H}^{\ell+1}$ to $\mathcal{H}^{\ell}$. Then, we refine it by using a novel $K$-way refinement heuristic which takes the primary and the secondary metrics into account. The proposed heuristic runs in multiple passes where in a pass it visits each boundary vertex $u$ and either leaves it in part $[u]$, or moves it to another part according to some move selection policy.

UMPa provides refinement methods for four primary metrics: the total communication volume TV; the maximum send volume MSV; the total number of messages TM; and the maximum number of messages a processing unit sends MSM. We use the notation $\mathrm{UMPa}_{X}$ to denote the partitioner with the primary objective function $X$ during the refinement. These methods take secondary and sometimes tertiary metrics into accounts. As an aid to the refinement process, the part weights can be used for tie-breaking purposes while selecting the best move.

Since each metric is different, the implementation details of the refinement heuristics are also different. However, their main logic is the same. The heuristics perform a number of passes on the boundary vertices. To be precise, UMPa uses at most $2^{\ell}$ passes for $\mathcal{H}^{\ell}$, the hypergraph at the $\ell$ th coarsening level. We observed that most of the improvement on the metrics are coming from the refinement on the coarser hypergraphs. Furthermore, since these hypergraphs are smaller, the passes on them take much less time. That is, the impact of these passes are high and their overhead is low. Hence, we decided to perform more passes on the coarser hypergraphs. UMPa also stops the passes when the improvement on the primary metric is not significant during a pass.

The high-level structure of a pass is given in Algorithm 1. In a pass, the heuristic visits the boundary vertices in a random order and for each visited vertex $u$ and for all $p \neq$ part $[u]$, it computes how the communication metrics in $\left(M_{1}, M_{2}, M_{3}\right)$ are affected when $u$ is moved from part $[u]$ to $p$. This computation 


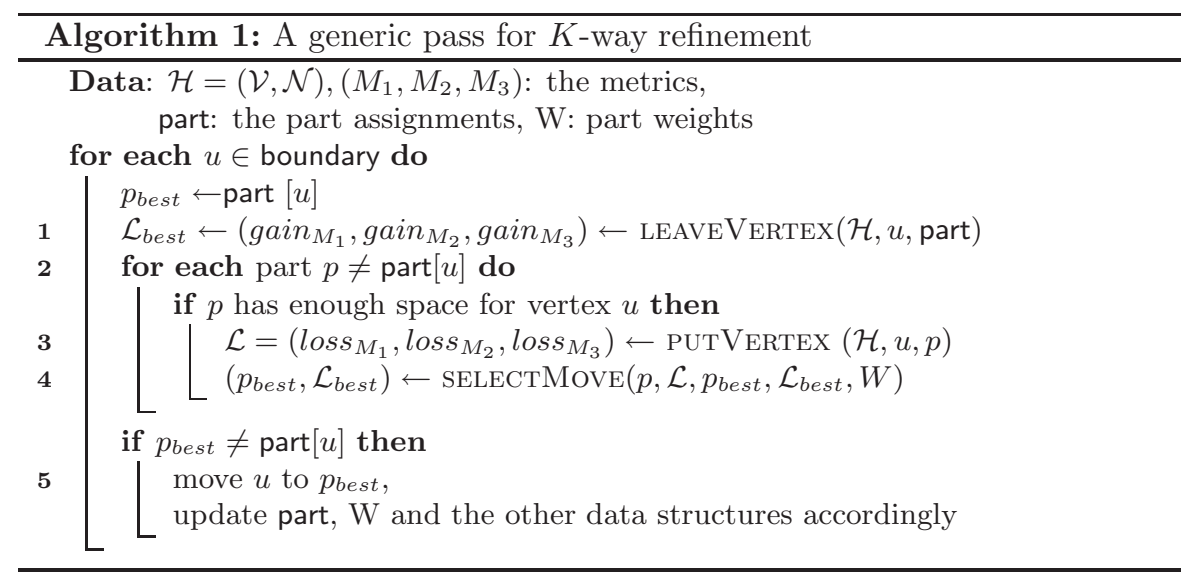

is realized in two steps. First, $u$ is removed from part $[u]$ and the leave gains for the communication metrics are computed with LEAVEVERTEX (line 1). Second, $u$ is tentatively put into a candidate part $p$ and the arrival losses are calculated with PutVertex (line 3). We first set the processing unit for the best move as $p_{\text {best }}=\operatorname{part}[u]$. Since the leave gains and arrival losses are equal while removing a vertex from its part and putting it back (the total gain is zero), initially, the best arrival loss triplet $\mathcal{L}_{\text {best }}$ is set to $\left(\right.$ gain $_{M_{1}}$, gain $_{M_{2}}$, gain $\left._{M_{3}}\right)$. Then, for each possible target processing unit $p$, the arrival losses on the metrics are computed. With the SELECTMOVE heuristic, which is given in Algorithm 2, these losses are compared with $\mathcal{L}_{\text {best }}$ to select the best move (line 4 ). After trying all target processing units and computing the arrival losses of the corresponding moves, $u$ is moved to $p_{\text {best }}$. If $p_{\text {best }} \neq \operatorname{part}[u]$ the data structures, such as boundary, part, $\lambda$, and $\Lambda$, used throughout the partitioning process are updated accordingly.

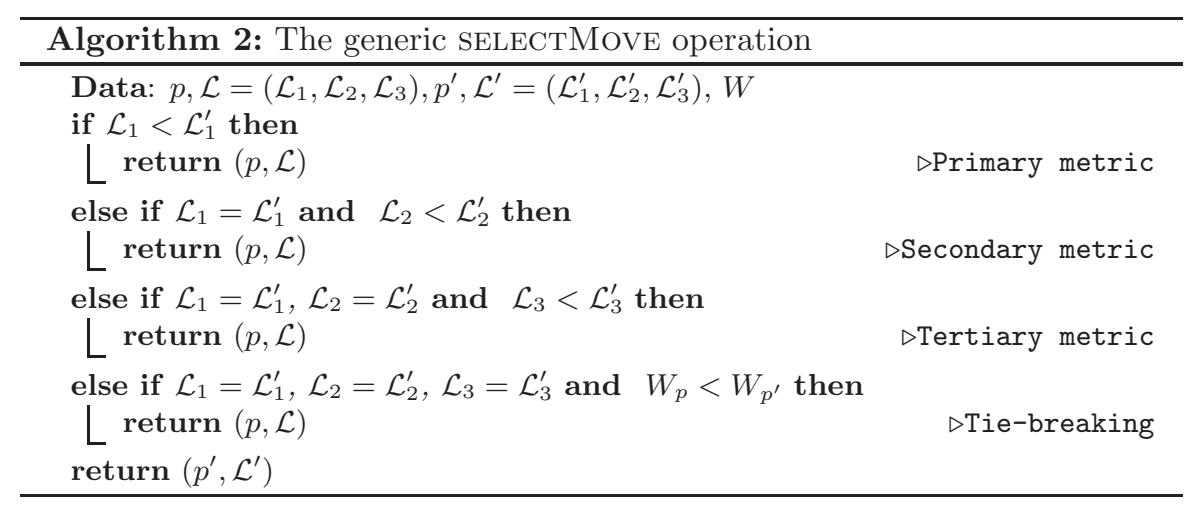

The SELECTMOVE heuristic given in Algorithm 2 compares two vectors $\left(\mathcal{L}_{1}, \mathcal{L}_{2}, \mathcal{L}_{3}, W_{p}\right)$ and $\left(\mathcal{L}_{1}^{\prime}, \mathcal{L}_{2}^{\prime}, \mathcal{L}_{3}^{\prime}, W_{p^{\prime}}\right)$. Starting from the first ones, it compares 
the vector entries one by one. When a smaller entry is found, the corresponding processing unit is returned as the better candidate.

\section{Multi-objective one-phase $K$-way Refinement}

Although the structure of a pass and the execution logic is the same, the computation of the leave gains and arrival losses differ with respect to the communication metrics used. Here we describe the implementation of the functions LEAVEVERTEX and PUTVERTEX for UMPaTV, UMPamsv with secondary and tertiary metrics MSRV and TV, and UMPaTM with a secondary metric TV. We also implemented UMPamsm and used it in the experiments. But we do not give the details of its leave gain and arrival loss computations since the structure of these algorithms is similar to the ones described below.

\subsection{Total communication volume}

In the directed hypergraph model, the total volume of the communication sent/received throughout the execution corresponds to the cutsize definition (2.2) as in the standard hypergraph model. In other words, the TV metric is global and hence, the sense of direction does not have any effect in its computation. Here we describe UMPaTv which takes a single communication metric TV into account, i.e., it is a standard hypergraph partitioner with the traditional objective function (2.2).

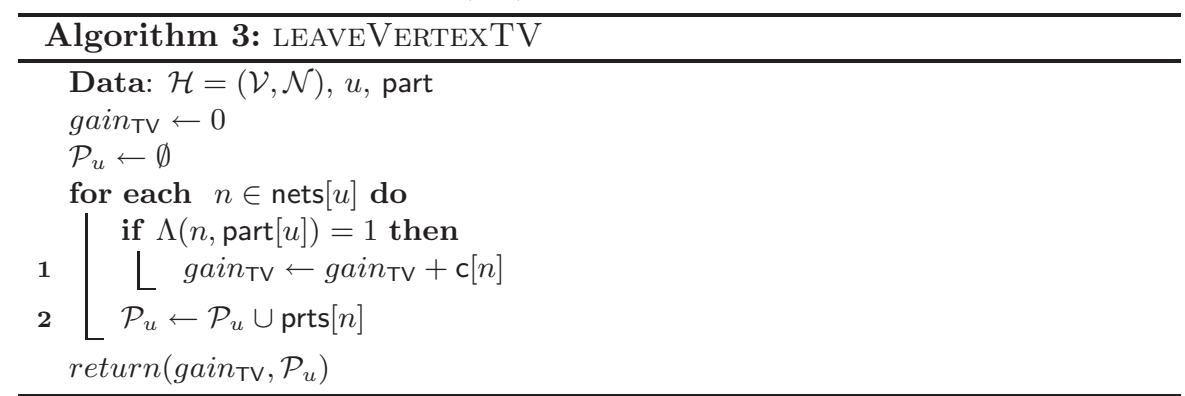

As described above, the refinement heuristic performs passes on the boundary vertices. Let $u$ be the boundary vertex visited during a pass. The LEAVEVERTEXTV method given in Algorithm 3 computes the gain on TV when $u$ leaves part $[u]$. After the move, there will be a gain if and only if the target processor $p$ contains at least one vertex which shares a net with $u$. Let $\mathcal{P}_{u}$ be the set of such parts. To be more efficient, this set can be computed beforehand and the for loop in Algorithm 1 (line 2) can be iterated over $p \in \mathcal{P}_{u}$ instead of $p \in\{1, \ldots, K\} \backslash$ part $[u]$. For this reason, LEAVEVERTEXTV also builds this set (line 2) while computing gain TV. $_{\text {. }}$

After $u$ leaves part $[u]$ and the leave gain is computed, the refinement heuristic computes the arrival losses for all target parts $\mathcal{P}_{u} \backslash$ part $[u]$. Algorithm 4 gives the description of the PUTVERTEXTV function. Given $u$ and a part $p$, the function computes the increase on TV after putting $u$ to $p$. For each net $n \in \operatorname{nets}[u]$, TV increases by $\mathrm{c}[n]$ if $u$ will be the only pin of $n$ in $p$ (line 1 ). 


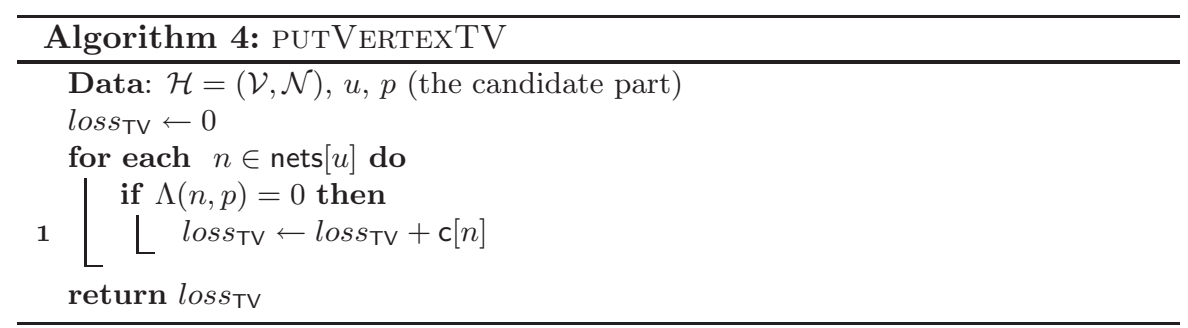

\subsubsection{Total number of messages}

Although the directionality is not important for the total volume, the flow of communication is crucial while minimizing other metrics. Hence for each net $n$ affected by a move, we need to use the source information $\operatorname{src}[n]$. Here we describe the leave gain and arrival loss computations of UMPaTM which minimizes the total number of messages throughout the execution by also taking the total volume of communication, TV, into account as the secondary metric.

Let $n e t \operatorname{Comm}\left(p, p^{\prime}\right)$ be the number of nets $n$ such that $p, p^{\prime} \in \operatorname{prts}[n]$ and $\operatorname{src}[n]=p$, i.e., the number of data to be sent from the processing unit $p$ to the processing unit $p^{\prime}$. The refinement heuristic performs passes on the boundary vertices. Let $u$ be the boundary vertex visited during a pass. When $u$ leaves part $[u]$, the gains on TM and TV are computed by LEAVEVERTEXTM given in Algorithm 5. The gain on TV (line 1) is computed as described in the previous subsection. For the gain on TM, there are two cases:

1. $u$ is the source pin of a net $n$ (line 2): For each part $p \in \operatorname{prts}[n]$, there is a gain if netComm $(\operatorname{part}[u], p)=0$ after removing $u$ from part $[u]$. Note that a vertex can be a source for multiple nets.

2. $u$ is a non-source pin of a net $n$ (line 4$)$ : Let $p_{\text {sender }}$ be the part having the source of $n$. There is a gain if netComm $\left(p_{\text {sender }}, \operatorname{part}[u]\right)=0$ after removing $u$ from part $[u]$.

In addition to gain computations, the set of the parts that receive data from $u$ (i.e., $u$ is the source of a net connected to the receiver part), receivers, and send data to $u$ (i.e., $u$ is a non-source pin of a net whose source is in the sender part), senders, are obtained. These sets are used to efficiently compute the arrival losses for each target part candidate.

After the leave gains are computed with LEAVEVERTEXTM, the heuristic tentatively moves the boundary vertex to the candidate parts and computes the losses on the communication metrics with PUTVERTEXTM given in Algorithm 6. Let $p$ be the target part. The function first computes the arrival loss on TV as described in the previous subsection. Then for all $p_{\text {receiver }} \in$ receivers, it checks if a new message from $p$ to $p_{\text {receiver }}$ is necessary. This implies a loss on TM. It repeats a similar process for all $p_{\text {sender }} \in$ senders and checks if a message from $p_{\text {sender }}$ to $p$, which was not necessary before the move, is now necessary. 

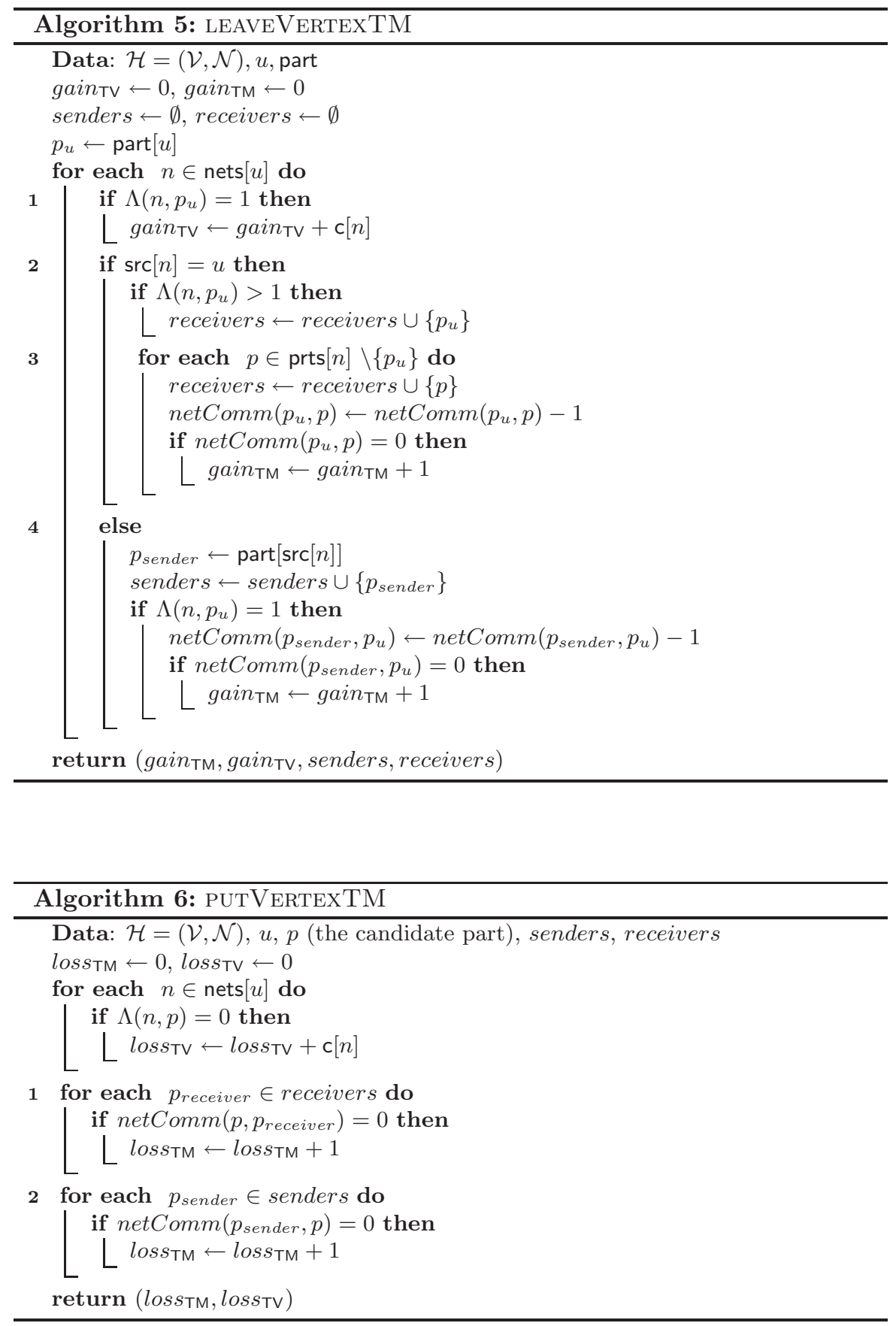


\subsubsection{Maximum communication volume}

Here we describe UMPamsv which minimizes MSV, the maximum volume sent by a processing unit, and also takes MSRV (the maximum send and receive volume), and TV into account as the secondary and tertiary metrics, respectively. This metric configuration is useful when one wants a partition which does not yield a bottleneck processor and does not use the network extensively.

The generic structure of the refinement heuristic given in Algorithm 1 also applies to the local communication metrics such as MSV and MSM. But for an efficient implementation, we slightly alter this structure for UMPaMsv. When a vertex is taken from its part and put to another one during the refinement, instead of computing the exact gains/losses on the metrics, we compute a set of intermediate values which correspond to the changes in the send/receive volumes of a processing unit $p$, i.e., $\mathrm{SV}[p]$ and $\mathrm{RV}[p]$. After each move, these intermediate values are used to compute the exact changes on the metrics, and the best move is selected in a similar fashion.

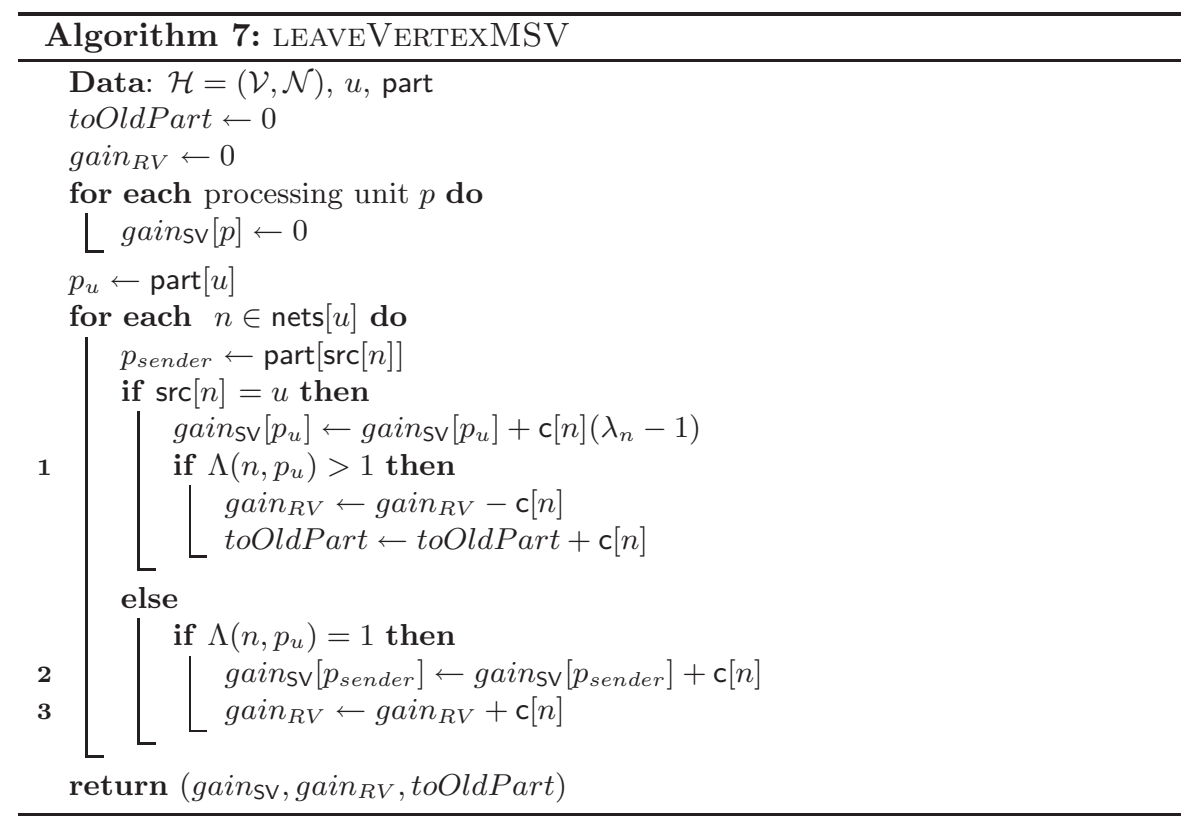

Algorithm 7 describes the LEAVEVERTEXMSV function. To store the changes on the send volumes of the processing units after the leave operation, the function uses an array gain $_{\mathrm{SV}}[\cdot]$ of size $K$. It also stores the change on RV[part[u]], i.e., the receive volume of part $[u]$, in a variable $\operatorname{gain}_{R V}$. If $u$ is the source for a net $n$, removing $u$ yields $\mathrm{c}[n]\left(\lambda_{n}-1\right)$ gain on the send volume of part $[u]$. But after the move, there will also be an increase on $\mathrm{RV}[\operatorname{part}[u]]$ if there are more pins of $n$ in part $[u]$ (line 1 ). The potential increase due to the nets having $u$ as the source does not depend on the target part. It is stored in a variable 
toOldPart which later will be used by the PUTVERTEXMSV function to compute the overall change on the send volume of each target part. When $u$ leaves $\operatorname{part}[u], \mathrm{RV}[\operatorname{part}[u]]$ decreases by $\mathrm{c}[n]$ if $u$ is not the source of $n$ and if it is the only pin of $n$ in part $[u]$ (line 2). The same decrease must also be observed on $\mathrm{SV}\left[p_{\text {sender }}\right]$, the send volume of the part containing $u$ 's source pin (line 3 ).

If $u$ is the source pin of $n$, as described above, we take the gain on SV [part $[u]]$ into account. However, we do not do the same for the corresponding gains on the $\mathrm{RV}$ values of other parts in prts $[n]$. That is, we do not compute all the reductions on RV, and hence, if we compute the metrics here the MSRV value will not be exact. Although exact RV values and hence the exact gains on the metrics can be computed, we chose to follow this approach to be more efficient. Because, after putting $u$ to the target part $p$, these reductions on the receive volumes of the processing units in $\operatorname{prts}[n] \backslash\{\operatorname{part}[u], p\}$ will not be effective anymore since these processing units will continue to receive $n$ 's data from $p$. Hence, if one computes the exact RV values here most of the computation will be redundant.

The details of the PUTVERTExMSV function are given in Algorithm 8. Similar to LEAVEVERTEXMSV, the algorithm computes the increase on the send volumes of all parts and the increase on the receive volume of the target part $p$. After the move, the target processing unit will send the data which was sent by part $[u]$ before. Hence, the value $\operatorname{loss}_{\mathrm{SV}}[p]$ must cover gain Sv $\left[p_{u}\right]$. It also needs to contain the amount of the communication from $p$ to part $[u]$ due to the nets having $u$ as the source that remain connected to part $[u]$, i.e., toOldPart (line 1). The rest of the loss updates are similar to the ones in the previous algorithms.

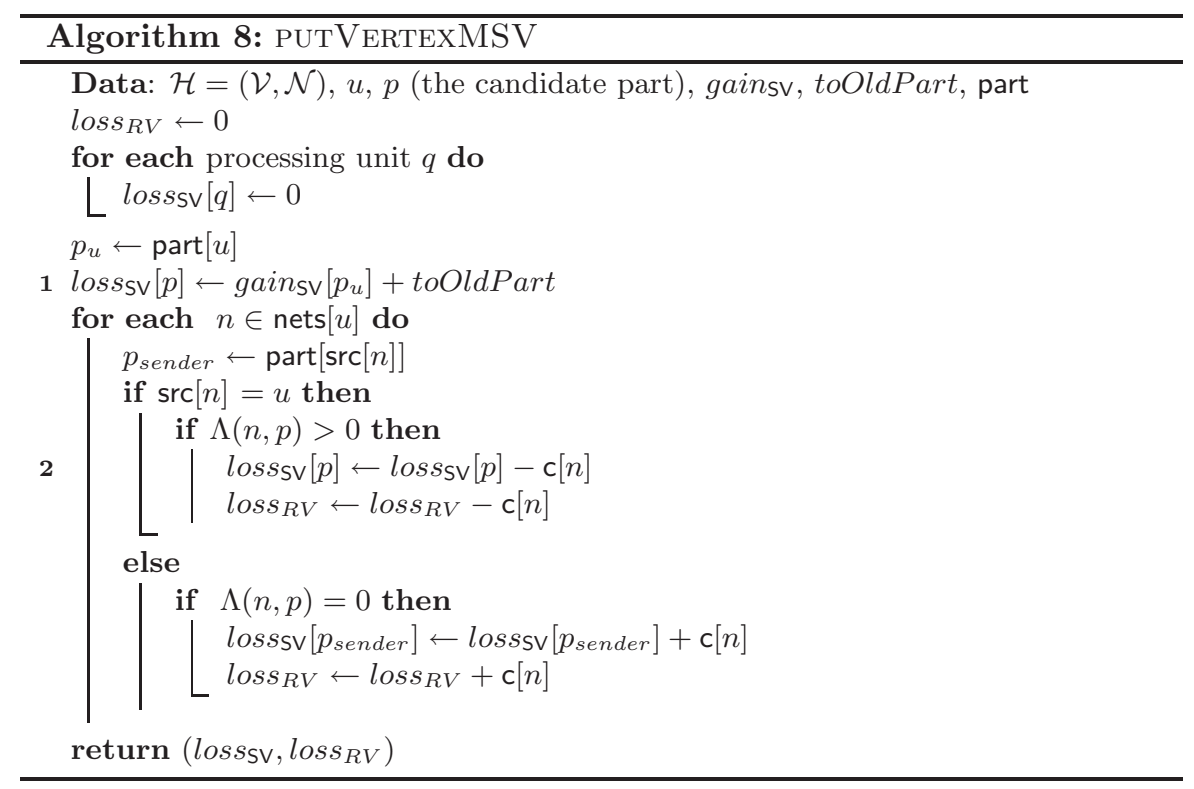

Algorithm 9 shows a pass of the proposed refinement heuristic of UMPaMSV. 
Firstly, $u$ is removed from part $[u]$, and the intermediate values, partial gains on the send/receive volumes, are computed by LEAVEVERTEXMSV. Then, $u$ is tentatively put to each target candidate $p$, and the intermediate values, arrival losses on the send/receive volumes, are obtained by PUTVERTEXMSV. As described above, although these intermediate values are inexact, their sum gives the exact change on the send/receive volumes for each processor. After computing these changes and the send/receive volumes after the move, the metrics are computed and the best move is selected in a similar fashion. That is the move with the smallest value on the primary metric is preferred. If these values are equal for two target processors, then the secondary metric is considered. In case of equality for the secondary metric values, the tertiary metric is taken into account. As we will show in the experimental results section, this move selection policy and tie-breaking scheme have positive impact on all the communication metrics.

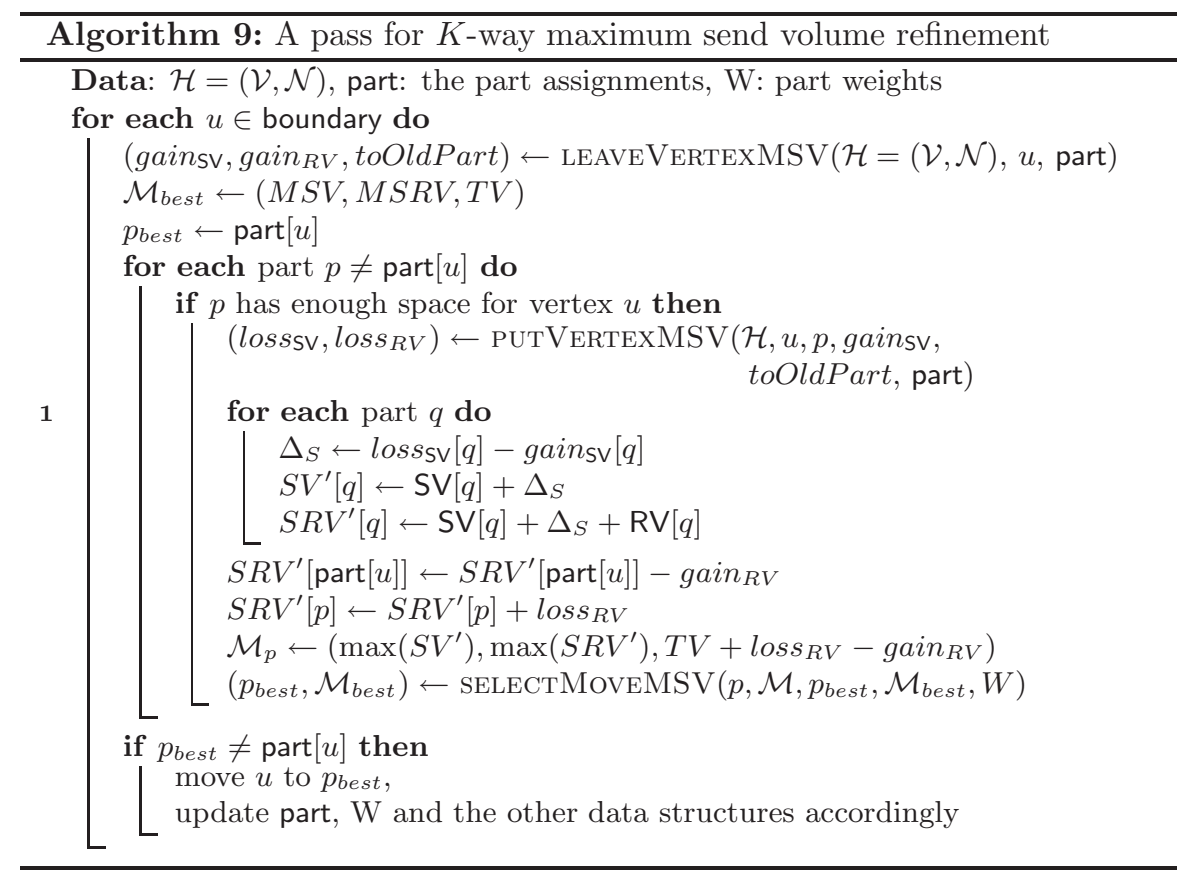

\subsubsection{Implementation Details}

During the gain computations, the heuristic uses the connectivity information between the nets and parts stored in data structures $\lambda, \Lambda$, and netComm. These structures are constructed after the initial partitioning phase and maintained by the uncoarsening phase. When a vertex $u$ is moved, we revisit the nets of $u$ and update the data structures accordingly. When new boundary vertices are detected, they are inserted to the array boundary and visited in the same pass. We restrict the number of moves for a vertex $u$ during a pass to 4 , in order to limit the execution time. When this number is reached the vertex is 
locked and removed from the boundary.

Let $\rho=\sum_{n \in \mathcal{N}}|\operatorname{pins}[n]|$ be the number of pins in a hypergraph. For UMPaTV, considering the restriction on the number of moves per vertex, the time complexity of the leave gain and arrival loss computations is $\mathcal{O}(\rho)$ and $\mathcal{O}(\rho K)$, respectively. Hence, the overall complexity of a refinement pass is $\mathcal{O}(\rho K)$. The factor $K$ comes from the number of candidate target parts for each move. For UMPaTV, we only visit the boundary vertices and try the parts of the visited vertex's nets as target candidates. In practice, these numbers are much smaller than $|\mathcal{V}|$ and $K$ as a side-effect of the minimization of the objective functions.

For UMPaтм, UMPamsv, and UMPamsm, the overall time complexity of a refinement pass is $\mathcal{O}\left(\rho K+|\mathcal{V}| K^{2}\right)$ since all these variants need an additional for loop which iterates $\mathcal{O}(K)$ times (lines 1 and 2 in Algorithm 6 and line 1 in Algorithm 9). However, since only the boundary vertices and parts of the nets are visited, the factors are much smaller in practice, and the worst-case analysis is very loose.

To store the numbers of pins per part for each net, $\Lambda$, we use a 2-dimensional array. Hence, the space complexity is $\mathcal{O}(K|\mathcal{N}|)$. This can be improved by using a sparse storage as shown in [18].

\section{Experimental results}

The experiments are conducted on a computer with $2.27 \mathrm{GHz}$ dual quad-core Intel Xeon CPUs and 48GB main memory.

We compare the quality and efficiency of the partitioners UMPa, Zoltan [12], Mondriaan [11] (version 4.0) and $\mathrm{PaToH}[10]$ (in default setting) ${ }^{1}$. UMPa is implemented in $\mathrm{C}++$, while the other partitioners are implemented in C. All implementations are compiled with the gcc suite version 4.4.4. To obtain our data set, we used several graphs with $100 \mathrm{~K}-1,500 \mathrm{~K}$ vertices and 409K-38, 354K edges from the 10th DIMACS implementation challenge dataset [35] which contains 38 graphs from eight different classes. The names and the details of these graphs are given in Table 1. These graphs are translated into hypergraphs using the column-net model to fit the problem definition of the 10th DIMACS challenge ${ }^{2}$. In all of our experiments, the vertices have unit weights, while the nets may have non-uniform costs (as in the ComputationalTask graphs). Although Zoltan hypergraph partitioner is designed to work on distributed memory machines, all executions are run serially in our experiments. The reported results are the averages of five different executions. The performances of the partitioners are evaluated for five different communication metrics in addition to execution time. These are the maximum send volume (MSV), the maximum send/receive volume (MSRV), the total volume (TV), the maximum send message (MSM), and

\footnotetext{
${ }^{1}$ The default setting of $\mathrm{PaToH}$ is chosen instead of quality setting, as the quality option obtains only $4 \%$ better cut sizes. On the other hand, the default option is 3.56 times faster.

2 http://www.cc.gatech.edu/dimacs10/data/dimacs10-rules
} 
the total message (TM). The chosen hypergraphs are partitioned into 128, 256, 512 , and 1024 parts with $\varepsilon=0.03$.

We first compare the execution time and the quality of the existing hypergraph partitioning tools. Although Mondriaan uses a hypergraph model, the partitioner is specifically designed for matrices, and it does not accept nonuniform net weights. For this reason, the experiment is run on 36 (out of 38) graphs excluding the ones in the ComputationalTask class. We adjust Mondriaan as described in [36] for the experiment. Figure 3 gives the communication metrics and execution times of Zoltan and Mondriaan normalized w.r.t. PaToH. As seen in the figure, Mondriaan obtains partitions whose TV values are similar to that of $\mathrm{PaToH}$ on TV. However, its quality on the other metrics is slightly worse. Furthermore, it is significantly slower than PaToH. Although the partitions found by Zoltan yield similar communication metrics when $K$ is small, Zoltan's partitioning quality gets worse as $K$ increases. We attribute this to the simplified refinement implementations for the parallelization purposes. Since PaToH obtains the best quality and the best serial execution time, we compare the performance of UMPa against $\mathrm{PaToH}$ for the rest of the experiments. A fair comparision between UMPa and existing work $[7,8]$ is not feasible due to the different partitioning contexts, as noted before (in Sections 2.4 and 3.1).

Figures 4 and 5 show the average values of the metrics normalized with respect to the corresponding average metric value of $\mathrm{PaToH}$ partition. Each column of the table (and its visual representation as a group of five bars) corresponds to an UMPa variant with a different metric and tie-breaking combination, while each row of the table corresponds to the values with respect to a different communication metric. We experimented with four main variants UMPax with the primary metric $X \in\{T V, M S V, T M, M S M\}$. For each of these variants, we tried different combinations where in the first one only the primary metric is taken into account with no tie-breaking. Then, except for UMPaTV, we obtained a variant which use additional metrics (shown with a ' + ' sign in the figures). At last, we added tie-breaking and obtained the full variant (shown with "+ PW" in the figures). We executed each of these 11 variants and PaToH on all 38 hypergraphs 5 times and obtain 5 different partitions. For each variant/metric pair, the geometric mean of these 190 executions are computed, and the averages for the UMPa variants are normalized with respect to PaToH's averages. The figures show these normalized values.

As the first two columns in the tables (and the first two bar groups) of the figures show, for various $K$ values, UMPaTv is as good as $\mathrm{PaToH}$ for all the communication metrics. Furthermore, its MSV and MSRV (2nd and 3rd rows/bars) values are $4-8 \%$ better than $\mathrm{PaToH}$ for all $K$ values. The tie-breaking scheme (the second column denoted with PW), which uses the part weight information, improves the performance of UMPaTV (the first column) by around $2 \%$. Since the direction information is not important while minimizing TV, the undirected hypergraph model and recursive bisection can be used as is. Therefore, the proposed directed hypergraph model and the $K$-way partitioning do not have an advantage for TV unlike for the other communication metrics as we discuss below. 


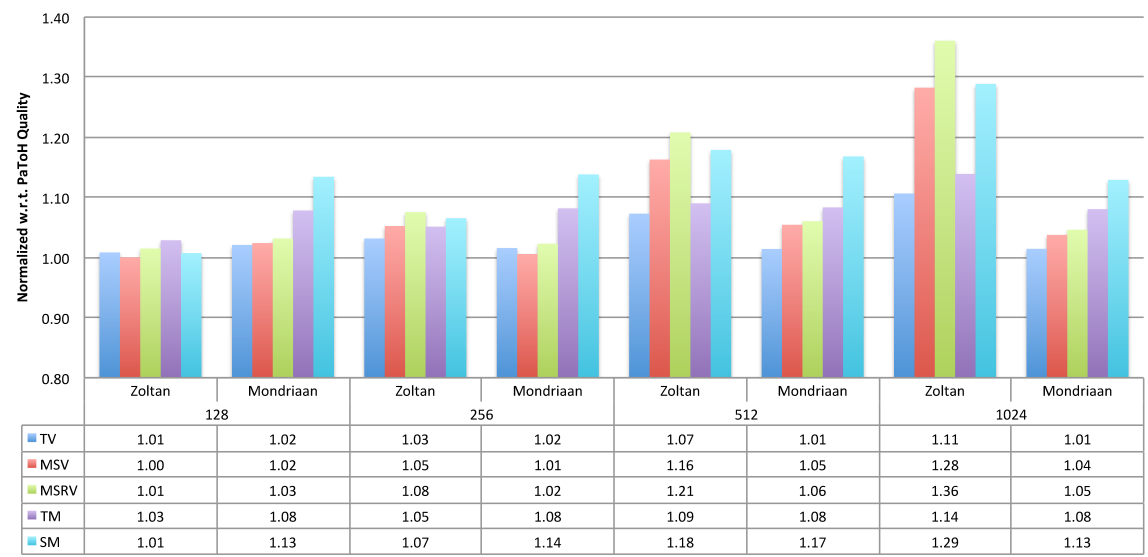

(a) Normalized communication metrics

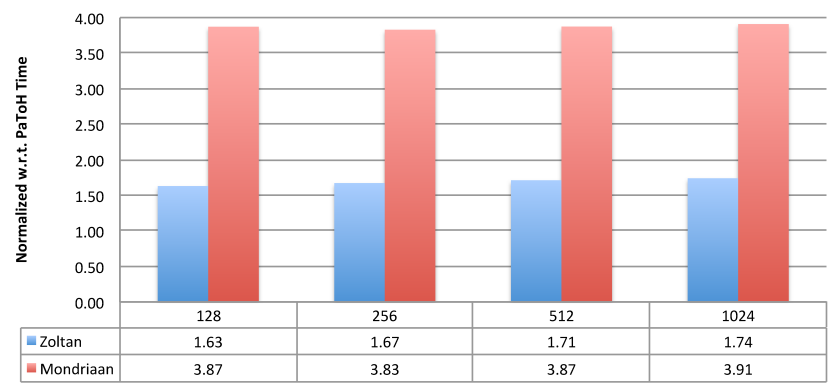

(b) Normalized execution times

Figure 3: The normalized communication metrics and execution times of Zoltan and Mondriaan w.r.t. PaToH for $K \in\{128,256,512,1024\}$.

We experimented with three UMPamsv variants to carefully evaluate the effects of the secondary and tertiary objectives and the tie-breaking mechanism. Although minimizing only with the primary metric and no tie-breaking (column 3) obtains $7-11 \%$ better MSV value, with respect to the other metrics, UMPamsv is worse than PaToH. Using MSRV and TV as the secondary and tertiary objectives (column 4) has positive effects on the other communication metrics. Moreover, this approach further improves the primary communication metric MSV. This variant of UMPamsv doubles the improvement on the primary metric MSV and reduces it $17-22 \%$ on the average. Furthermore, it also obtains decent values for the other metrics with respect to PaToH. Extending the refinement with part weight tie-breaking (column 5) also reduces all the volume-based communication metrics around $2 \%$. These observations are not only true on the average but also for most of the graphs in the dataset. For all partitionings with different $K$ values, UMPamsv with tie-breaking obtains more than 20\% improvement in MSV for 12 graphs. The improvement is between $10 \%$ 
and $20 \%$ for 13 graphs, and between $2 \%$ to $10 \%$ for the remaining 13 graphs.

Only with a primary metric, UMPaTM with no tie-breaking (column 6) improves TM about $7-12 \%$. It also improves MSM with respect to $\mathrm{PaToH}$, but its effect is almost always negative for the volume-based metrics. Using TV as the secondary objective to UMPатм (column 7) reduces the increase on these metrics. Furthermore, it also improves TM by 2-3\% more. Similar to other cases, the communication metrics are improved around $3 \%$ more with the addition of tie-breaking (column 8).

The results for UMPaMSM are similar to that of other variants. Only with the primary objective function (column 9 ), UMPamsM obtains $5-7 \%$ better MSM values than $\mathrm{PaToH}$. Using TM and TV as the secondary and tertiary objectives (column 10) makes the difference around 7-19\%. A further 2-3\% improvement is obtained with the addition of tie-breaking (column 11).

Figure 6 shows the UMPa variants' runtimes normalized with respect to $\mathrm{Pa}$ ToH. As a $K$-way partitioner, the complexity of the refinement is linear with the number of parts for UMPaтv and UMPaтM, and quadratic for UMPamsv and UMPamsm. Therefore, as $K$ increases, the proposed direct $K$-way method is expected to be slower than a recursive-bisection-based partitioner. For 128 parts, except UMPamsv, the execution times are closer to that of $\mathrm{PaToH}$. However, when the number of parts get bigger, the slowdown increases.

Table 1 gives the individual execution times and the volume metric results of PaToH for $K=512$. The normalized execution times and normalized volume metrics of the UMPa variants are listed on the right side of the table. As seen in the table, the best TV improvement of the UMPaTV is $18 \%$ improvement on G n pin pout, while it obtains 5\% TV degrade on eu-2005. UMPamsv obtains its best MSV improvement on preferentialAttachment as $85 \%$, while it obtains its worst improvement on thermal2 graph by $1 \%$. UMPатм and UMPaмsm have their best improvements on smallworld as $45 \%$ and $43 \%$, respectively.

As Table 1 shows, the Citation and Clustering graphs have relatively large MSV, TM, and MSM values compared to other classes. For these graphs, the UMPa variants obtain decent improvements. However, when these metrics have small values, e.g., PaToH's MSM values for the Sparse class, the improvements are not significant. Since PaToH minimizes only the total communication volume, a small metric value implies that minimizing TV also minimizes that metric. We believe that this is due to the structure of the graphs. For example, the Delaunay graphs are planar. Hence, when $\mathrm{PaToH}$ puts similar vertices in the same part, the nets connected to a part will have similar pin sets. Even if some of these nets are in the cut, the corresponding communications can be packed into a few messages. The same argument is also true for the Random Geometric graphs where only the vertices close to each other in the Euclidian space are connected.

\section{Conclusions and future work}

We proposed a directed hypergraph model and a multi-level partitioner UMPa. The partitioner uses a novel $K$-way refinement heuristic employing 


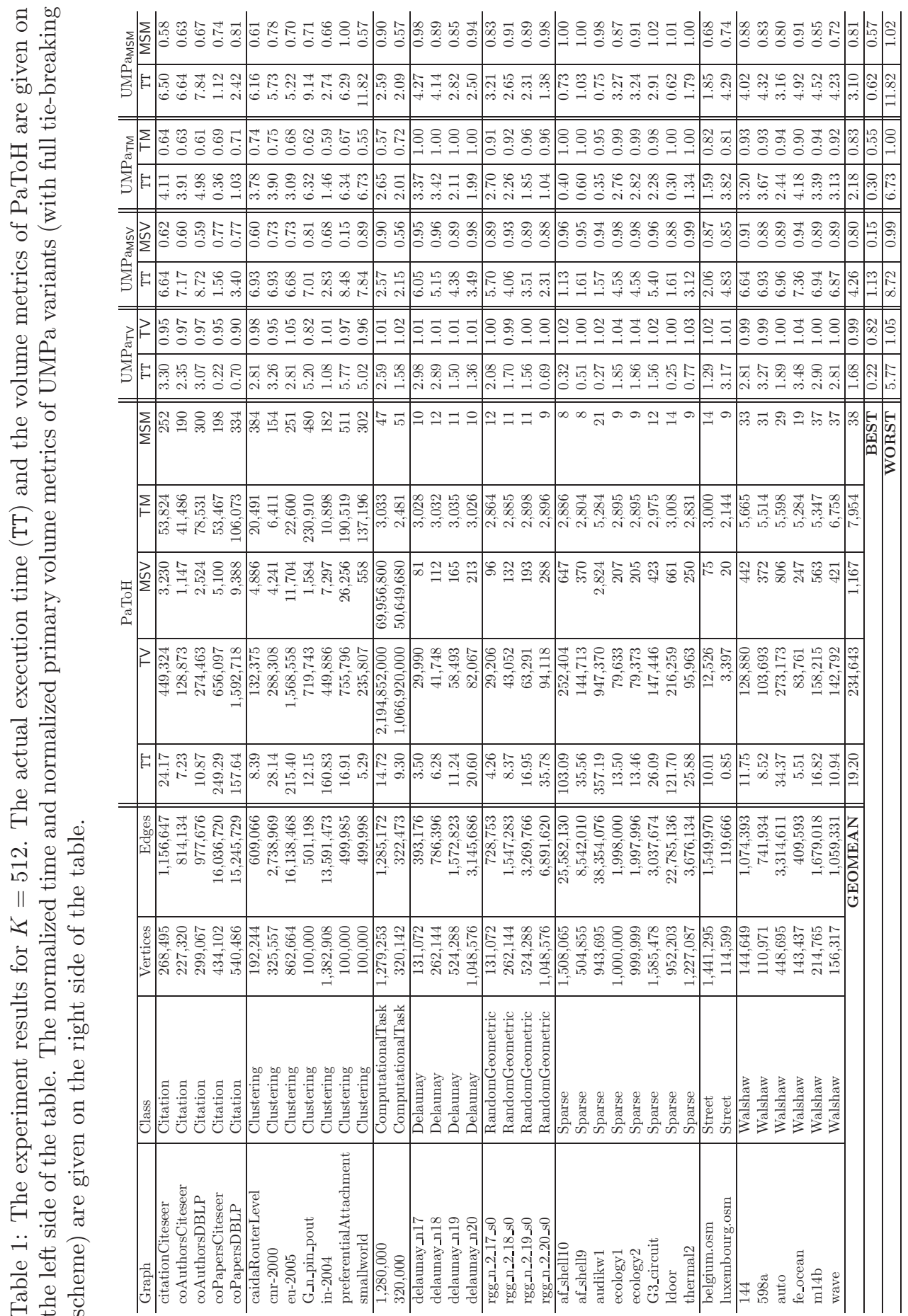




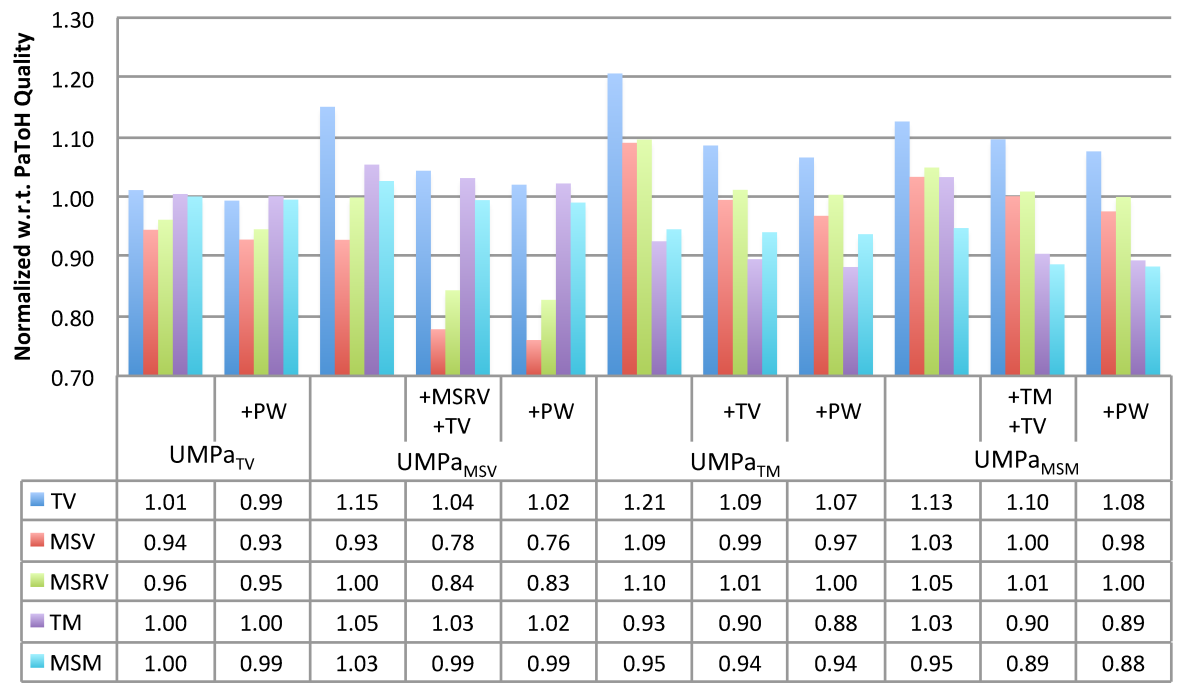

(a) $K=128$

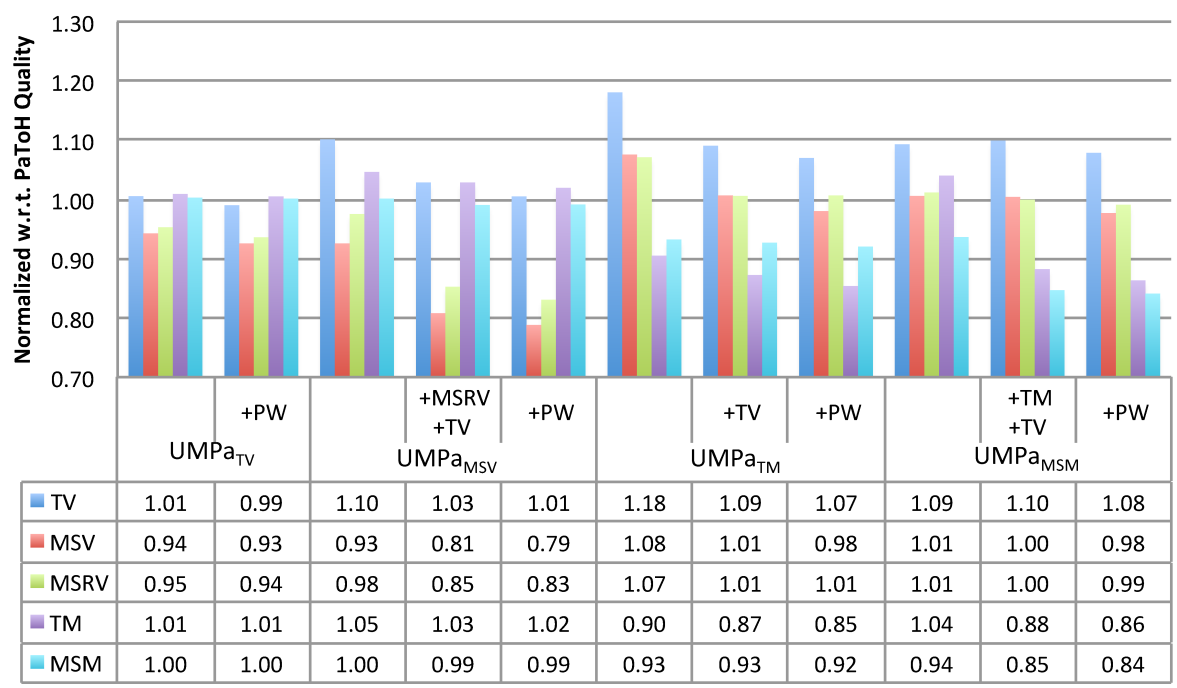

(b) $K=256$

Figure 4: The normalized metrics of UMPa w.r.t. PaToH for $K=128$ and $K=256$.

a tie-breaking scheme to handle multiple communication metrics. UMPa yields good communication patterns by reducing multiple communication metrics all together.

Although most of the previous research has mainly focused on the minimization of the total communication volume, there are studies on the minimization of 


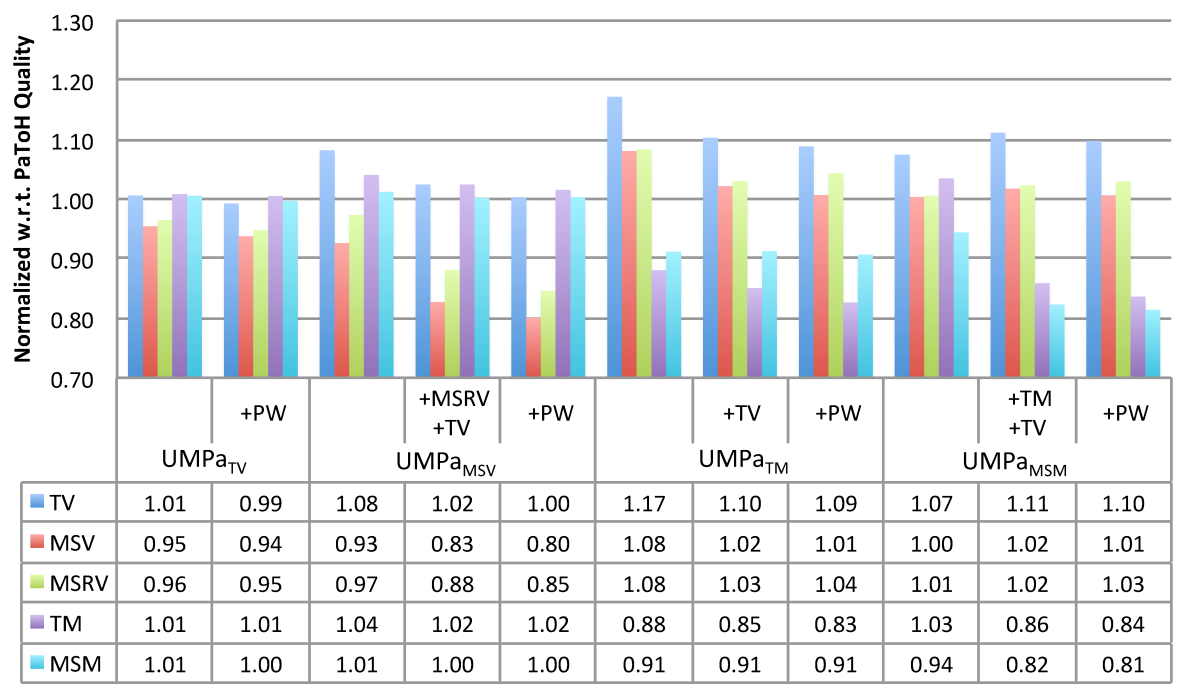

(a) $K=512$

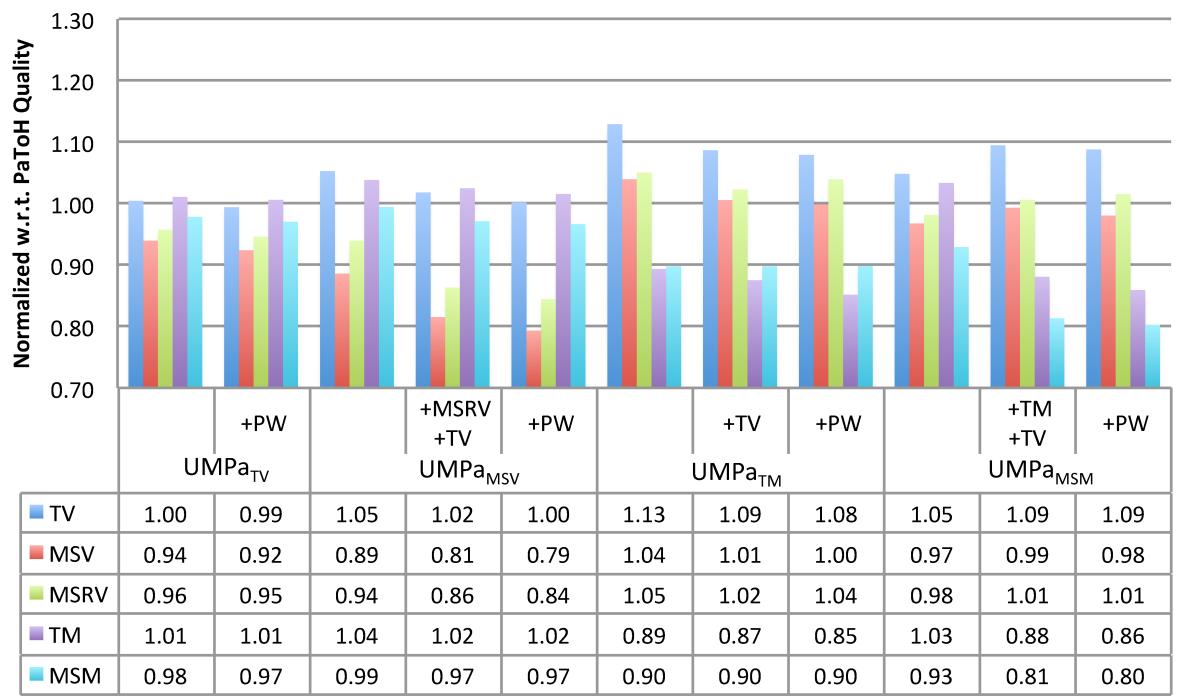

(b) $K=1024$

Figure 5: The normalized metrics of UMPa w.r.t. $\mathrm{PaToH}$ for $K=512$ and $K=1024$.

multiple metrics. Existing hypergraph-based solutions on multiple metrics follow a two-phase approach where in each phase a different metric is minimized, sometimes at the expense of others. UMPa follows a one-phase approach where all the communication cost metrics are effectively minimized in a multi-objective 


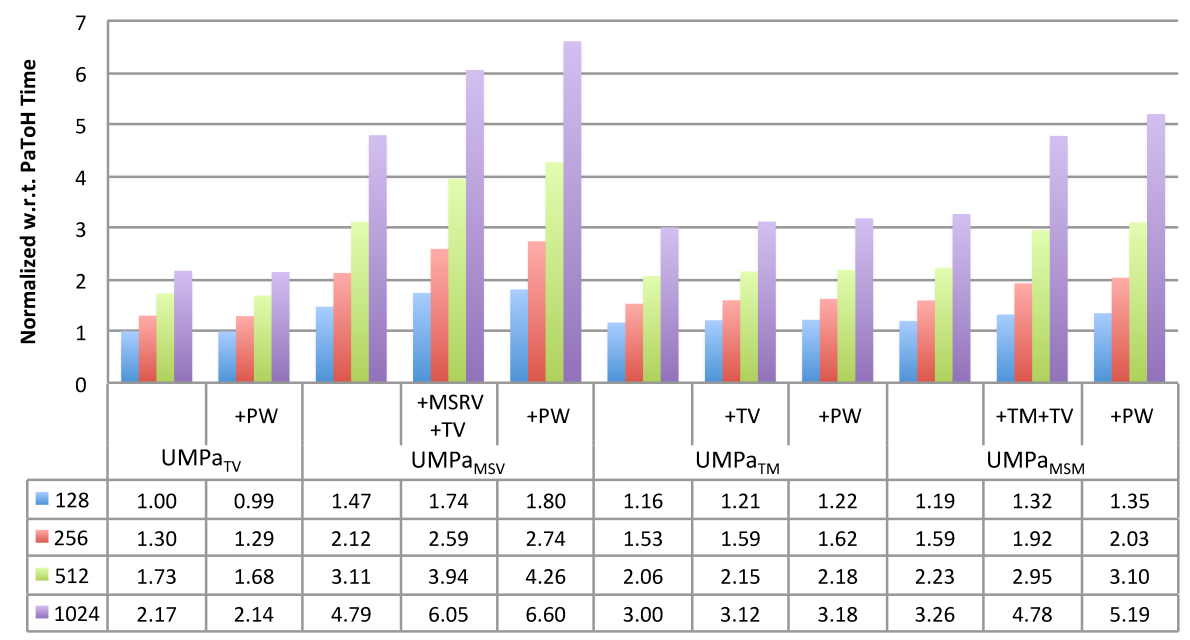

Figure 6: The normalized execution times of UMPa w.r.t. PaToH.

setting where reductions in all metrics can be achieved at the same time, thanks to the proposed directed hypergraph model (undirected models were discussed to be inadequate). We conducted experiments with large hypergraphs and up to 1024 parts in which the proposed approach yielded improvements over the state-of-the-art hypergraph partitioner PaToH. For example, for $K=1024$, UMPamsm produces $20 \%$ and $14 \%$ better partitions in terms of MSM and TM, respectively.

We plan to speed up UMPa and the proposed refinement approach by implementing them on modern parallel architectures. Investigating the hypergraph partitioning models and communication metrics for hierarchical memory systems, such as clusters of multi-socket, multi-core machines with accelerators is another direction of research. We plan to extend UMPa to handle the directed hypergraphs with multiple senders. Such directed hypergraph models can be used to model computations which require communications for outputs or for both inputs and outputs. Columnwise or nonzero-based sparse matrix partitioning models yield such directed hypergraphs.

\section{Acknowledgment}

This work was supported in parts by the DOE grant DE-FC02-06ER2775; by the NSF grants CNS-0643969, OCI-0904809 and OCI-0904802; and by France ANR project SOLHAR (ANR-13-MONU-0007).

\section{References}

[1] B. Hendrickson, R. Leland, An improved spectral graph partitioning algorithm for mapping parallel computations, SIAM Journal on Scientific 
Computing 16 (2) (1995) 452-469.

[2] Ü. V. Çatalyürek, C. Aykanat, Hypergraph-partitioning based decomposition for parallel sparse-matrix vector multiplication, IEEE Transactions on Parallel and Distributed Systems 10 (7) (1999) 673-693.

[3] Ü. V. Çatalyürek, C. Aykanat, B. Uçar, On two-dimensional sparse matrix partitioning: Models, methods, and a recipe, SIAM Journal on Scientific Computing 32 (2) (2010) 656-683.

[4] B. Hendrickson, T. G. Kolda, Graph partitioning models for parallel computing, Parallel Computing 26 (2000) 1519-1534.

[5] C. Walshaw, M. G. Everett, M. Cross, Parallel dynamic graph partitioning for adaptive unstructured meshes, Journal of Parallel and Distributed Computing 47 (1997) 102-108.

[6] D. M. Pelt, R. H. Bisseling, A medium-grain method for fast 2d bipartitioning of sparse matrices, in: 28th IEEE International Parallel and Distributed Processing Symposium, 2014.

[7] B. Uçar, C. Aykanat, Encapsulating multiple communication-cost metrics in partitioning sparse rectangular matrices for parallel matrix-vector multiplies, SIAM J. Sci. Comput. 25 (2004) 1837-1859.

[8] R. H. Bisseling, W. Meesen, Communication balancing in parallel sparse matrix-vector multiplication, Electronic Transactions on Numerical Analysis 21 (2005) 47-65.

[9] Ü. V. Çatalyürek, M. Deveci, K. Kaya, B. Uçar, UMPa: A multi-objective, multi-level partitioner for communication minimization, in: 10th DIMACS Implementation Challenge Workshop: Graph Partitioning and Graph Clustering, 2012, pp. 53-66, published in Contemporary Mathematics, Vol. 588, Editors D.A. Bader, H. Meyerhenke, P. Sanders, D. Wagner, 2013.

[10] Ü. V. Çatalyürek, C. Aykanat, PaToH: A Multilevel Hypergraph Partitioning Tool, Version 3.0, Bilkent University, Department of Computer Engineering, Ankara, 06533 Turkey. PaToH is available at http://bmi.osu.edu/ umit/software.htm (1999).

[11] B. Vastenhouw, R. H. Bisseling, A two-dimensional data distribution method for parallel sparse matrix-vector multiplication, SIAM Review 47 (1) (2005) 67-95.

[12] K. Devine, E. Boman, R. Heaphy, R. Bisseling, Ü. V. Çatalyürek, Parallel hypergraph partitioning for scientific computing, in: Proceedings of 20th International Parallel and Distributed Processing Symposium (IPDPS), IEEE, 2006. 
[13] T. Lengauer, Combinatorial Algorithms for Integrated Circuit Layout, Wiley-Teubner, Chichester, U.K., 1990.

[14] T. N. Bui, C. Jones, A heuristic for reducing fill-in in sparse matrix factorization, in: Proc. 6th SIAM Conf. Parallel Processing for Scientific Computing, SIAM, 1993, pp. 445-452.

[15] S. T. Barnhard, H. D. Simon, Fast multilevel implementation of recursive spectral bisection for partitioning unstructured problems, Concurrency: Practice and Experience 6 (2) (1994) 67-95.

[16] B. Hendrickson, R. Leland, A multilevel algorithm for partitioning graphs, in: Proc. Supercomputing, ACM, New York, NY, USA, 1995, p. 28.

[17] A. Trifunovic, W. Knottenbelt, Parkway 2.0: A parallel multilevel hypergraph partitioning tool, in: Proc. ISCIS, Vol. 3280 of LNCS, Springer Berlin / Heidelberg, 2004, pp. 789-800.

[18] C. Aykanat, B. B. Cambazoglu, B. Uçar, Multi-level direct k-way hypergraph partitioning with multiple constraints and fixed vertices, Journal of Parallel and Distributed Computing 68 (5) (2008) 609-625.

[19] B. W. Kernighan, S. Lin, An efficient heuristic procedure for partitioning graphs, The Bell System Technical Journal 49 (2) (1970) 291-307.

[20] C. M. Fiduccia, R. M. Mattheyses, A linear-time heuristic for improving network partitions, in: Proc. 19th Design Automation Conference, 1982, pp. $175-181$.

[21] Ü. V. Çatalyürek, C. Aykanat, A fine-grain hypergraph model for 2D decomposition of sparse matrices, in: Proceedings of 15th International Parallel and Distributed Processing Symposium (IPDPS), San Francisco, CA, 2001.

[22] B. Uçar, C. Aykanat, Minimizing communication cost in fine-grain partitioning of sparse matrices, in: A. Yazici, C. Şener (Eds.), Computer and Information Sciences - ISCIS 2003, Vol. 2869 of Lecture Notes in Computer Science, Springer Berlin / Heidelberg, 2003, pp. 926-933.

[23] B. Uçar, C. Aykanat, Revisiting hypergraph models for sparse matrix partitioning, SIAM Review 49 (4) (2007) 595-603.

[24] Y.-K. Kwok, I. Ahmad, Static scheduling algorithms for allocating directed task graphs to multiprocessors, ACM Computing Surveys 31 (4) (1999) 406-471.

[25] L. Valiant, A bridging model for parallel computation, Comm. of the ACM 33 (8) (1990) 103-111.

[26] R. H. Bisseling, Parallel Scientific Computation: A Structured Approach Using BSP and MPI, Oxford, 2004. 
[27] R. Ferreira, T. Kurc, M. Beynon, C. Chang, A. Sussman, J. Saltz, Objectrelational queries into multi-dimensional databases with the Active Data Repository, Parallel Processing Letters 9 (2) (1999) 173-195.

[28] J. Dean, S. Ghemawat, MapReduce: Simplified data processing on large clusters, Communications of ACM 51 (1) (2008) 107-113.

[29] J. Ekanayake, H. Li, B. Zhang, T. Gunarathne, S.-H. Bae, J. Qiu, G. Fox, Twister: a runtime for iterative MapReduce, in: The First International Workshop on MapReduce and its Applications (MAPREDUCE'10) - HPDC2010, 2010, pp. 810-818.

[30] T. Condie, N. Conway, P. Alvaro, J. M. Hellerstein, K. Elmeleegy, R. Sears, MapReduce online, in: Proceedings of the 7th USENIX conference on Networked systems design and implementation, NSDI'10, USENIX Association, Berkeley, CA, USA, 2010, pp. 21-21.

[31] G. Gallo, G. Longo, S. Pallottino, S. Nguyen, Directed hypergraphs and applications, Discrete Appl. Math. 42 (2-3) (1993) 177-201.

[32] Ü. V. Çatalyürek, C. Aykanat, A hypergraph-partitioning approach for coarse-grain decomposition, in: ACM/IEEE SC2001, Denver, CO, 2001.

[33] M. Ravishankar, J. Eisenlohr, L.-N. Pouchet, J. Ramanujam, A. Rountev, P. Sadayappan, Code generation for parallel execution of a class of irregular loops on distributed memory systems, in: Proceedings of the International Conference on High Performance Computing, Networking, Storage and Analysis, SC '12, 2012, pp. 72:1-72:11.

[34] C. J. Alpert, A. B. Kahng, Recent directions in netlist partitioning: A survey, VLSI Journal 19 (1-2) (1995) 1-81.

[35] 10th DIMACS implementation challenge: Graph partitioning and graph clustering, http://www.cc.gatech.edu/dimacs10/ (2011).

[36] R. H. Bisseling, B. O. Fagginger Auer, Abusing a hypergraph partitioner for unweighted graph partitioning, in: 10th DIMACS Implementation Challenge Workshop: Graph Partitioning and Graph Clustering, 2012, pp. 1936, published in Contemporary Mathematics, Vol. 588, Editors D.A. Bader, H. Meyerhenke, P. Sanders, D. Wagner, 2013. 


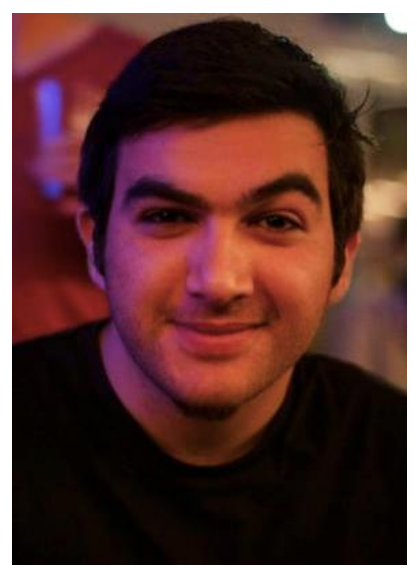

Mehmet Deveci is a PhD Student in the Department of Computer Science \& Engineering at The Ohio State University. His research interests are bioinformatics and high performance computing. He received his B.S. in Computer Engineering from Middle East Technical University, Turkey in 2010.

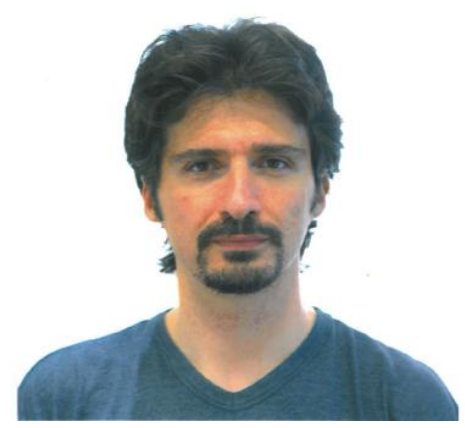

Kamer Kaya is a Assistant Professor in the Department of Biomedical Informatics at The Ohio State University. He received the PhD degrees (2009) in Computer Engineering from Bilkent University, Ankara, Turkey. His research deals with cryptography, parallel computing and algorithms.

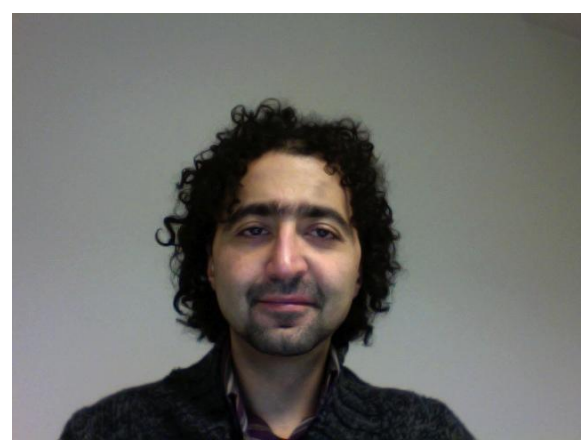

Bora Uçar is a CNRS researcher at LIP, ENS-LYON. He received the PhD degrees (2005) in Computer Engineering from Bilkent University, Ankara, Turkey. His research interests are combinatorial scientific computing and high performance computing. 


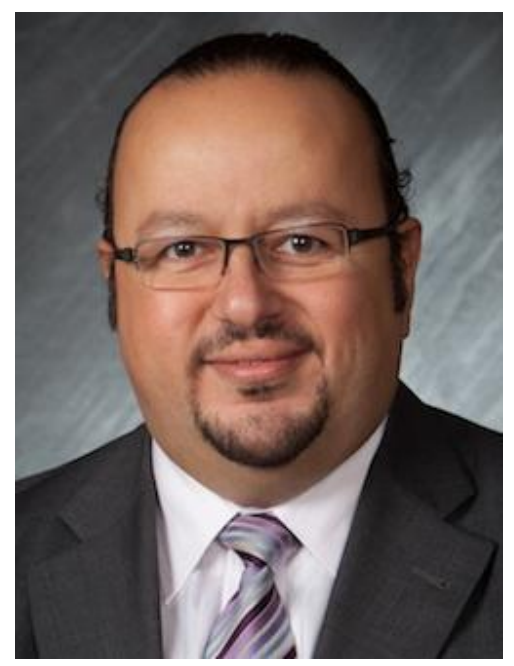

Umit V. Catalyurek is a Professor in the Department of Biomedical Informatics at The Ohio State University, and has a joint faculty appointment in the Department of Electrical and Computer Engineering. His research interests include combinatorial scientific computing, run-time systems for data-intensive computing, and highperformance computing in biomedicine. He received his Ph.D., M.S. and B.S. in Computer Engineering and Information Science from Bilkent University, Turkey, in 2000, 1994 and 1992, respectively. 